\title{
The functional moderate deviations for Harris recurrent Markov chains and applications
}

\author{
Xia Chen ${ }^{\mathrm{a}, 1}$, Arnaud Guillin ${ }^{\mathrm{b}}$ \\ ${ }^{a}$ Department of Mathematics, University of Tennessee, Knoxville, TN 37996-1300, USA \\ b CEREMADE, Université Paris Dauphine, pl. Mar. de Lattre de Tassigny, 75775 Paris cedex 16, France
}

Received 17 January 2003; accepted 15 July 2003

\begin{abstract}
We study exponentially asymptotic behaviors for the trajectories of additive functionals of Harris Markov chains. In the main result, we establish a moderate deviation principle for a pair of additive functionals of different growth rates. Moreover, we give explicit formulas for the rate functions which exhibit a non-quadratic behavior. In particular, we achieve the functional moderate deviations in two different scales. As an application, we obtain a functional law of LIL, which leads to a variety of strong limit laws in the spirit of Strassen [Z. Wahr. Geb. 3 (1964) 211-226].

(C) 2003 Elsevier SAS. All rights reserved.

\section{Résumé}

Nous étudions dans cet article le comportement asymptotique des trajectoires de fonctionnelles additives de chaînes de Markov Harris récurrentes. Le principal résultat énonce le principe de déviations modérées d'un couple de fonctionnelles additives ayant différentes vitesses de croissance, et donc à deux échelles différentes. De plus, nous donnons les formes explicites des fonctions de taux, possédant typiquement un caractère non quadratique. En application, nous obtenons une loi fonctionnelle du logarithme itéré, qui conduisent, en suivant les idées de Strassen [Z. Wahr. Geb. 3 (1964) 211-226], à différentes lois limites fortes.

(c) 2003 Elsevier SAS. All rights reserved.
\end{abstract}

MSC: $60 \mathrm{~F} 10 ; 60 \mathrm{~F} 15 ; 60 \mathrm{~F} 17 ; 60 \mathrm{~J} 55$

Keywords: Harris recurrent Markov chain; p-regularity; Moderate deviation; The law of the iterated logarithm

E-mail address: guillin@ceremade.dauphine.fr (A. Guillin).

${ }^{1}$ Supported in part by NSF Grant DMS-0102238.

0246-0203/\$ - see front matter (c) 2003 Elsevier SAS. All rights reserved. doi:10.1016/j.anihpb.2003.07.002 


\section{Introduction and results on moderate deviations}

Let $\left\{X_{n}\right\}_{n} \geqslant 0$ be a Harris recurrent Markov chain with state space $(E, \mathcal{E})$, transition probability $P(x, A)$ and invariant measure $\pi$. Throughout, we always assume that the $\sigma$-algebra $\mathcal{E}$ is countably generated. By Harris recurrence we mean that $\left\{X_{n}\right\}_{n \geqslant 0}$ is irreducible and for any $A \in \mathcal{E}^{+}$and any initial distribution $\mu$,

$$
P_{\mu}\left\{X_{n} \in A \text { infinitely often }\right\}=1,
$$

where

$$
\mathcal{E}^{+}=\{A \in \mathcal{E} ; \pi(A)>0\}
$$

and the standard notation $P_{\mu}$ is for the Markovian probability with initial distribution $\mu$ (Naturally, $E_{\mu}$ is for the correspondent expectation; $P_{x}$ and $E_{x}$ for $P_{\mu}$ and $E_{\mu}$, respectively, as $\mu=\delta_{x}$ is a Dirac measure.). We introduce $P^{k}(x, A)$ for the $k$-step transition of $\left\{X_{n}\right\}_{n \geqslant 0}$. By Harris recurrence, for any $A \in \mathcal{E}^{+}$

$$
\tau_{A} \equiv \inf \left\{n \geqslant 1 ; X_{n} \in A\right\}<\infty \quad \text { a.s. }
$$

Our goal is to establish the moderate deviations for additive functionals of $\left\{X_{n}\right\}_{n} \geqslant 0$ mainly in the case of null recurrence (although some of our results also include the case of positive recurrence). The moderate deviations arise from the needs for tail control in the study of strong limit theorems such as the law of the iterated logarithm. See, for example, Chung and Hunt [11], Erdös and Taylor [19], Kesten [24], Jain and Pruitt [22], Révész [31], Marcus and Rosen [27], for the results in the context of recurrent Levy processes and random walks, and Touati [34], Wu [35], Csáki and Salminen [13], de Acosta [2], de Acosta and Chen [3], Gantert and Zeitouni [20], Chen [7-10], Guillin [21], Djellout and Guillin [17] for those in the case of recurrent Markov processes (use also Duflo et al. [5]). Among them, the probability estimate carried out for the law of the iterated logarithm given in Chen $[9,10]$ suggests the existence of the non-trivial limits for the sequences

$$
\frac{1}{b_{n}} \log P\left\{\sum_{k=1}^{n} f\left(X_{k}\right) \geqslant \sqrt{a\left(\frac{n}{b_{n}}\right)} b_{n}\right\}, \quad n=1,2, \ldots
$$

and

$$
\frac{1}{b_{n}} \log P\left\{\sum_{k=1}^{n} g\left(X_{k}\right) \geqslant a\left(\frac{n}{b_{n}}\right) b_{n}\right\}, \quad n=1,2, \ldots,
$$

where $\left\{b_{n}\right\}$ can be any positive sequence satisfying

$$
b_{n} \rightarrow \infty \quad \text { and } \quad b_{n} / n \rightarrow 0 \quad(n \rightarrow \infty),
$$

$a(\cdot)$ is the partial Green function given as below, $f$ is centered with respect to the invariant measure $\pi, g \geqslant 0$ and $f, g$ satisfy some regularity conditions. An important special case is the case of local times in which $\left\{X_{n}\right\}_{n} \geqslant 1$ is 1 or 2 dimensional discrete random walk and, the functions $g$ and $f$ are, respectively, the indicator of a single point and the difference between two such indicators (see (1.22) and (1.23) below for an example). On the other hand, one will see in the later of this section that as the Markov chain is positive recurrent or is close to be positively recurrent, the tail behaviors of the second sequence can not determine full large (moderate) deviation.

We study, at the level of moderate deviations, the asymptotic sample path properties of the additive functionals generated by $f$ and by $g$, together with asymptotic correlation between these two different types of additive functionals. To this end, we first introduce some concepts from the book by Revuz [33].

A bounded, non-negative Borel-measurable function $g$ on $(E, \mathcal{E})$ is called a special function if

$$
\sup _{x \in E} E_{x} \sum_{k=1}^{\tau_{A}} g\left(X_{k}\right)<+\infty \quad \forall A \in \mathcal{E}^{+} .
$$


Given a Harris recurrent Markov chain, the class of special functions is dense in the function spaces such as $L^{p}(E, \mathcal{E}, \pi)(p \geqslant 1)$. When the Markov chain takes discrete values, any function with finite support is special. In particular, local times are generated by special functions. For the Markov chain satisfying certain continuity in Feller sense, a typical special function is a function with compact support. Also, if $P$ is quasi-compact, every bounded positive function is special.

A special function is $\pi$-integrable. Indeed, we may choose a $A \in \mathcal{E}^{+}$such that $\pi(A)<+\infty$. Therefore,

$$
\pi(g) \equiv \int g(x) \pi(d x)=(\pi(A))^{-1} \int_{A} \pi(d x) E_{x} \sum_{k=1}^{\tau_{A}} g\left(X_{k}\right) \leqslant \sup _{x \in A} E_{x} \sum_{k=1}^{\tau_{A}} g\left(X_{k}\right)<+\infty .
$$

A Borel-measurable function $f$ on $(E, \mathcal{E})$ is called a charge if $|f|$ is special and $\pi(f)=0$. By Theorem 1.5 in Chen [9], for any charge $f$,

$$
\int f^{2}(x) \pi(d x)<\infty \text { and } \sum_{k=1}^{\infty} \int f(x) P^{k} f(x) \pi(d x) \text { converges. }
$$

Consequently, the energy $\sigma_{f}^{2}$ given by

$$
\sigma_{f}^{2}=\int f^{2}(x) \pi(d x)+2 \sum_{k=1}^{\infty} \int f(x) P^{k} f(x) \pi(d x)
$$

is well defined for a charge $f$. In general, $\sigma_{f}^{2} \geqslant 0$. To make our case interesting, throughout we assume that $\sigma_{f}^{2}>0$.

In Orey [30], a set $D \in \mathcal{E}^{+}$is called $D$-set if its indicator $I_{D}$ is special. For a fixed $D$-set $D$, write the partial Green function

$$
a(t)=\frac{1}{\pi(D)} \sum_{k=1}^{[t]} v P^{k}(D) \quad(t \geqslant 1),
$$

where $v$ is a fixed probability measure on $(E, \mathcal{E})$. By recurrence $a(t) \uparrow \infty$ as $t \rightarrow \infty$. According to Theorem 2 , Chapter 2 in Orey [30], the asymptotic magnitude (as $t \rightarrow \infty)$ of $a(t)$ is independent of the choice of $D$ and $\nu$. Recall (Chen [8]) that a Harris Markov chain $\left\{X_{n}\right\}_{n \geqslant 0}$ is called $p$-regular, if $a(t)$ is regularly varying at $\infty$ with index $p$ :

$$
\lim _{t \rightarrow \infty} \frac{a(\lambda t)}{a(t)}=\lambda^{p} \quad \forall \lambda>0 .
$$

Clearly, $0 \leqslant p \leqslant 1$ if above equality holds. Note that every positive recurrent Markov chain is 1-regular. The concept of regular Markov chain is closely related to Hypothesis (C) introduced in Touati [34]. In the "atomic" case, the $p$-regularity with $0<p<1$ is equivalent to the asymptotic stability condition on the hitting time of an atom, which was introduced by Csáki and Csörgö [12].

By convention, $C[0,1]$ is the space of continuous functions on $[0,1]$ and $D[0,1]$ is the space of the functions on $[0,1]$ which are right continuous and have left limits on $[0,1]$. Under the uniform convergence topology, $C[0,1]$ and $D[0,1]$ become Banach spaces. We define $C\left\{[0,1], \mathbf{R}^{2}\right\}=C[0,1] \times C[0,1]$ and $D\left\{[0,1], \mathbf{R}^{2}\right\}=$ $D[0,1] \times D[0,1]$.

Throughout, we assume $f$ is a charge and $g$ is a special function. Define $D[0,1]$-valued random sequences $\left\{\xi_{n}(\cdot)\right\}$ and $\left\{\eta_{n}(\cdot)\right\}$ as

$$
\begin{aligned}
& \xi_{n}(t)=\sum_{k=1}^{[n t]} f\left(X_{k}\right), \quad 0 \leqslant t \leqslant 1, n=1,2, \ldots, \\
& \eta_{n}(t)=\sum_{k=1}^{[n t]} g\left(X_{k}\right), \quad 0 \leqslant t \leqslant 1, n=1,2, \ldots
\end{aligned}
$$


We shall study the moderate deviations associated with the $D\left\{[0,1], \mathbf{R}^{2}\right\}$-valued random sequence $\left\{\boldsymbol{\Xi}_{n}\right\}$ defined by

$$
\Xi_{n}(t)=\left(\frac{1}{\sqrt{a\left(n b_{n}^{-1}\right)} b_{n}} \xi_{n}(t), \frac{1}{a\left(n b_{n}^{-1}\right) b_{n}} \eta_{n}(t)\right), \quad 0 \leqslant t \leqslant 1
$$

where $n=1,2, \ldots$ and $\left\{b_{n}\right\}$ is a positive sequence satisfying (1.2).

Define the function $\Lambda_{p}^{*}(x, y)$ on $(-\infty, \infty) \times[0, \infty)$ :

$$
\Lambda_{p}^{*}(x, y)= \begin{cases}\frac{\pi(g) x^{2}}{2 \sigma_{f}^{2} y}+(1-p)\left(\frac{p^{p} y}{\pi(g) \Gamma(p+1)}\right)^{(1-p)^{-1}} & y>0, \\ 0 & x=y=0 \\ +\infty & \text { else. }\end{cases}
$$

One can verify that $\Lambda_{p}^{*}$ is lower semi-continuous and convex. Let

$$
\begin{array}{r}
\Theta_{p}=\left\{(\phi, \gamma) \in C\left\{[0,1], \mathbf{R}^{2}\right\} ;\right. \\
\phi(0)=\gamma(0)=0, \phi(t), \gamma(t) \text { are absolutely continuous, } \\
\left.\gamma(t) \text { is non-decreasing and } \int_{0}^{1} \Lambda_{p}^{*}(\dot{\phi}(t), \dot{\gamma}(t)) d t<\infty\right\} .
\end{array}
$$

Set

$$
I_{p}(\phi, \gamma)= \begin{cases}\int_{0}^{1} \Lambda_{p}^{*}(\dot{\phi}(t), \dot{\gamma}(t)) d t & \text { if }(\phi, \gamma) \in \Theta_{p} \\ +\infty & \text { otherwise }\end{cases}
$$

Theorem 1.1. Assume that the Harris recurrent Markov chain $\left\{X_{n}\right\}_{n} \geqslant 0$ is $p$-regular with $0<p<1$ and that $f$ is a charge, $g$ is special. Then for any initial distribution $\mu,\left\{\Xi_{n}\right\}$ satisfies the following moderate deviation principle: for each closed set $\mathcal{C} \in D\left\{[0,1], \mathbf{R}^{2}\right\}$

$$
\limsup _{n \rightarrow \infty} \frac{1}{b_{n}} \log P_{\mu}\left\{\Xi_{n} \in \mathcal{C}\right\} \leqslant-\inf _{(\phi, \gamma) \in \mathcal{C}} I_{p}(\phi, \gamma)
$$

and, for each open set $\mathcal{O} \in D\left\{[0,1], \mathbf{R}^{2}\right\}$

$$
\liminf _{n \rightarrow \infty} \frac{1}{b_{n}} \log P_{\mu}\left\{\Xi_{n} \in \mathcal{O}\right\} \geqslant-\inf _{(\phi, \gamma) \in \mathcal{O}} I_{p}(\phi, \gamma)
$$

where the partial Green function $a(\cdot)$ is given in (1.5), and $\left\{b_{n}\right\}$ can be any positive sequence satisfying (1.2).

The moderate deviations for i.i.d. sequences has a long history (see, e.g., Dembo and Shao [14] for the type of self-renormalization and Jiang, Rao, Wang and Li [23] for deterministic normalization). Theorem 1.1 exhibits a non-standard behavior in the moderate deviations as the rate functions obtained here are not quadratic. 
By the canonical projection $(\phi, \gamma) \mapsto \gamma$ and by the contraction principle (see, e.g., Theorem 4.2.1 in Dembo and Zeitouni [15]), from Theorem 1.1 we obtain a moderate deviation principle for the additive functional generated by the special function $g$, with the rate function

$$
J_{p}(\gamma) \equiv \inf _{\phi \in D[0,1]} I_{p}(\phi, \gamma)= \begin{cases}(1-p) \int_{0}^{1}\left(\frac{p^{p} \dot{\gamma}(t)}{\pi(g) \Gamma(p+1)}\right)^{(1-p)^{-1}} d t & \text { if } \gamma \in \Gamma_{p} \\ +\infty & \text { otherwise }\end{cases}
$$

where

$\Gamma_{p}=\left\{\gamma \in D[0,1] ; \gamma(0)=0, \gamma(t)\right.$ is non-decreasing, absolutely continuous and $\left.\dot{\gamma} \in L^{(1-p)^{-1}}[0,1]\right\}$.

More precisely, we have

Theorem 1.2. Assume that the Harris recurrent Markov chain $\left\{X_{n}\right\}_{n} \geqslant 0$ is $p$-regular with $0<p<1$ and that $g$ is special. Then for any initial distribution $\mu$, the following moderate deviation principle holds: for each closed set $F \in D[0,1]$

$$
\limsup _{n \rightarrow \infty} \frac{1}{b_{n}} \log P_{\mu}\left\{\eta_{n}(\cdot) / a\left(n b_{n}^{-1}\right) b_{n} \in F\right\} \leqslant-\inf _{\gamma \in F} J_{p}(\gamma)
$$

and, for each open set $G \in D[0,1]$

$$
\liminf _{n \rightarrow \infty} \frac{1}{b_{n}} \log P_{\mu}\left\{\eta_{n}(\cdot) / a\left(n b_{n}^{-1}\right) b_{n} \in G\right\} \geqslant-\inf _{\gamma \in G} J_{p}(\gamma) .
$$

The case $p=1$ can not be included in Theorem 1.1 and Theorem 1.2, due to the singularity of the additive functional generated by $g$ in this situation. As a matter of fact, even at level of the simple moderate deviations, rate functions can be different from case to case. Although it is pointed out in Chen [10] that

$$
\lim _{n \rightarrow \infty} \frac{1}{b_{n}} \log P\left\{\sum_{k=1}^{n} g\left(X_{k}\right) \geqslant x a\left(\frac{n}{b_{n}}\right) b_{n}\right\}= \begin{cases}0 & \text { if } x<\pi(g), \\ -\infty & \text { if } x>\pi(g),\end{cases}
$$

as $p=1$, the full form of the moderate deviation for $\eta_{n}(1)$ is far more complicated. Indeed, if we take $\left\{X_{n}\right\}_{n} \geqslant 0$ as an i.i.d. sequence (in which case $\pi$ can be chosen as the common distribution of this sequence, all bounded measurable functions on $(E, \mathcal{E})$ are special, and $a(t) \sim t$ as $t \rightarrow \infty)$ then by the well known Cramér's large deviation principle (see, e.g., Theorem 2.2.3 in Dembo and Zeitouni [15]) for any $\varepsilon>0$, there is a $\delta>0$ such that

$$
P\left\{\left|\frac{1}{n} \sum_{k=1}^{n} g\left(X_{k}\right)-\pi(g)\right| \geqslant \varepsilon\right\} \leqslant \mathrm{e}^{-\delta n}
$$

for large $n$. Consequently, for every closed set $F$, and every open set $G$ in the line,

$$
\begin{aligned}
& \limsup _{n \rightarrow \infty} \frac{1}{b_{n}} \log P\left\{\eta_{n}(1) / a\left(n b_{n}^{-1}\right) b_{n} \in F\right\} \leqslant-\inf _{y \in F} l(y), \\
& \liminf _{n \rightarrow \infty} \frac{1}{b_{n}} \log P\left\{\eta_{n}(1) / a\left(n b_{n}^{-1}\right) b_{n} \in G\right\} \geqslant-\inf _{y \in G} l(y)
\end{aligned}
$$

as soon as $\left\{b_{n}\right\}$ satisfies (1.2), where the rate function $l(y)$ is given by

$$
l(y)= \begin{cases}0 & \text { if } y=\pi(g), \\ +\infty & \text { otherwise }\end{cases}
$$


In Example 1.6 (the case $q=2$ ) of Chen [9], on the other hand, an 1-regular but null Harris recurrent integervalued Markov chain $\left\{X_{n}\right\}_{n} \geqslant 0$ is constructed, such that

$$
a(n) \equiv \sum_{k=1}^{n} P^{k}(0,0) \sim \frac{\pi^{2}}{6} \frac{n}{\log n} \quad(n \rightarrow \infty) .
$$

Define

$$
\tau_{0}(0)=0 \quad \text { and } \quad \tau_{0}(k+1)=\inf \left\{n>\tau_{0}(k) ; X_{n}=0\right\} \quad(k \geqslant 1) .
$$

Let $g=I_{0}$. Then for any $\varepsilon>0$,

$$
\begin{aligned}
P_{0}\left\{\eta_{n}(1) \leqslant \varepsilon \frac{n}{\log n}\right\} & =P_{0}\left\{\tau\left(\left[\frac{\varepsilon n}{\log n}\right]\right) \geqslant n\right\} \geqslant P_{0}\left(\bigcup_{k=1}^{[\varepsilon n / \log n]}\left\{\tau_{0}(k)-\tau_{0}(k-1) \geqslant n\right\}\right) \\
& =1-\left(1-P_{0}\left\{\tau_{0} \geqslant n\right\}\right)^{[\varepsilon n / \log n]} \sim \frac{\varepsilon n}{\log n} P_{0}\left\{\tau_{0} \geqslant n\right\} .
\end{aligned}
$$

Notice that $E_{0} \tau_{0}=\infty$ and that $P\left\{\tau_{0} \geqslant n\right\}$ is regularly varying as $n \rightarrow \infty$. Trivially,

$$
\lim _{n \rightarrow \infty} \frac{1}{\log n} \log P_{0}\left\{\eta_{n}(1) \leqslant \varepsilon \frac{n}{\log n}\right\}=0 .
$$

In view of (1.12), one has a moderate deviation principle described in (1.13) and (1.14) with $b_{n}=\log n$ but with a different rate function given by

$$
l(y)= \begin{cases}0 & \text { if } y \leqslant \pi(g), \\ +\infty & \text { otherwise. }\end{cases}
$$

By considering the map $(\phi, \gamma) \mapsto \phi$ one can get a moderate deviation for $\xi_{n}(\cdot)$ governed by the rate function

$$
\kappa_{p}(\phi) \equiv \inf _{\gamma \in D[0,1]} I_{p}(\phi, \gamma)= \begin{cases}(2-p) \int_{0}^{1}\left(\frac{p^{p} \dot{\phi}^{2}(t)}{2 \Gamma(p+1) \sigma_{f}^{2}}\right)^{\frac{1}{2-p}} d t \quad \phi \in \Psi_{p}, \\ +\infty & \text { otherwise, }\end{cases}
$$

where

$$
\Psi_{p}=\left\{\phi \in C[0,1] ; \phi(0)=0, \phi(t) \text { is absolutely continuous and } \dot{\phi} \in L^{2(2-p)^{-1}}[0,1]\right\}
$$

and the equality in (1.15) can be seen from the simple fact that

$$
\inf _{y>0} \Lambda_{p}^{*}(x, y)=(2-p)\left(\frac{p^{p} x^{2}}{2 \Gamma(p+1) \sigma_{f}^{2}}\right)^{\frac{1}{2-p}}, \quad x \in \mathbf{R} .
$$

We can do slightly better. Indeed, the case $p=1$ can be included.

Theorem 1.3. Assume that the Harris recurrent Markov chain $\left\{X_{n}\right\}_{n \geqslant 0}$ is $p$-regular with $0<p \leqslant 1$ and that $f$ is a charge. Then for any initial distribution $\mu$, the following moderate deviation principle holds: for each closed set $F \in D[0,1]$

$$
\limsup _{n \rightarrow \infty} \frac{1}{b_{n}} \log P_{\mu}\left\{\xi_{n}(\cdot) / \sqrt{a\left(n b_{n}^{-1}\right)} b_{n} \in F\right\} \leqslant-\inf _{\phi \in F} \kappa_{p}(\phi)
$$

and, for each open set $G \in D[0,1]$

$$
\liminf _{n \rightarrow \infty} \frac{1}{b_{n}} \log P_{\mu}\left\{\xi_{n}(\cdot) / \sqrt{a\left(n b_{n}^{-1}\right)} b_{n} \in G\right\} \geqslant-\inf _{\phi \in G} \kappa_{p}(\phi) .
$$


A special case of $p=1$ is when $\left\{X_{n}\right\}_{n} \geqslant 0$ is positive recurrent, which means that the returning time to any $A \in \mathcal{E}^{+}$has finite expectation, or equivalently, the Markov chain has a finite invariant measure $\pi$. In this case we always make $\pi$ a probability measure by an appropriate normalization. Notice that by the law of large numbers, $a(t) \sim t$ as $t \rightarrow \infty$ and from (1.15)

$$
\kappa_{1}(\phi)= \begin{cases}\frac{1}{2 \sigma_{f}^{2}} \int_{0}^{1} \dot{\phi}^{2}(t) d t & \phi \in \Psi_{1}, \\ +\infty & \text { otherwise. }\end{cases}
$$

We have the following classic type of result on moderate deviations.

Corollary 1.4. Assume that the Markov chain $\left\{X_{n}\right\}_{n \geqslant 0}$ is positive recurrent and that $f$ is a charge. Then for any initial distribution $\mu$, the following moderate deviation principle holds: for each closed set $F \in D[0,1]$

$$
\limsup _{n \rightarrow \infty} \frac{1}{b_{n}} \log P_{\mu}\left\{\xi_{n}(\cdot) / \sqrt{n b_{n}} \in F\right\} \leqslant-\inf _{\phi \in F} \kappa_{1}(\phi)
$$

and, for each open set $G \in D[0,1]$

$$
\liminf _{n \rightarrow \infty} \frac{1}{b_{n}} \log P_{\mu}\left\{\xi_{n}(\cdot) / \sqrt{n b_{n}} \in G\right\} \geqslant-\inf _{\phi \in G} \kappa_{1}(\phi) .
$$

Remark 1.5. First of all, Theorems $1.1,1.2$ and 1.3 can easily be extended to the moderate deviations in $C\left\{[0,1], \mathbf{R}^{2}\right\}$ or $C[0,1]$ with $\xi_{n}$ and $\eta_{n}$ being replaced by their continuous versions:

$$
\begin{aligned}
& \xi_{n}^{c}(t)=\sum_{k=1}^{[n t]} f\left(X_{k}\right)+(n t-[n t]) f\left(X_{[n t]+1}\right), \quad 0 \leqslant t \leqslant 1, n=1,2, \ldots, \\
& \eta_{n}^{c}(t)=\sum_{k=1}^{[n t]} g\left(X_{k}\right)+(n t-[n t]) g\left(X_{[n t]+1}\right), \quad 0 \leqslant t \leqslant 1, n=1,2, \ldots,
\end{aligned}
$$

and with the same rate functions. It will be useful for applications in Section 5.

Remark 1.6. From the proof given in Section 3, we can see that the upper bounds given in Theorem 1.1 hold uniformly over initial points. So (1.7) can be strengthened into

$$
\limsup _{n \rightarrow \infty} \frac{1}{b_{n}} \log \sup _{x \in E} P_{x}\left\{\Xi_{n} \in \mathcal{C}\right\} \leqslant-\inf _{(\phi, \gamma) \in \mathcal{C}} I_{p}(\phi, \gamma)
$$

for every closed set $\mathcal{C} \in D\left\{[0,1], \mathbf{R}^{2}\right\}$. On the other hand, we do not expect such uniformity for the lower bounds, under the conditions given in Theorem 1.1.

Remark 1.7. Note that the case $p=0$ is not included in the above theorems. The main difficulty is that the random sequence $\left\{\Xi_{n}\right\}$ and its components fail to be exponentially tight in their value space as $p=0$. As a matter of fact, one can easily see that the level sets of the functions $I_{0}, J_{0}$ and $\kappa_{0}$ are not compact. However, the simple moderate deviations for $\Xi_{n}(1)$ in the case $p=0$ with rate functions $\Lambda_{0}^{*}$ are valid, by the same proof.

Application. We give here an application of our result to the additive functionals of recurrent random walks on the integer lattice $\mathbf{Z}$. Suppose that $S_{n}=\sum_{1}^{n} Y_{k}$ where $Y_{k}$ are iid symmetric integer-valued random variables with characteristic function

$$
\varphi(\lambda)=E \mathrm{e}^{i \lambda Y_{1}} .
$$


We assume that no proper subgroup of $\mathbf{Z}$ supports $\left\{S_{n}\right\}_{n} \geqslant 1$ and that $\left\{S_{n}\right\}_{n} \geqslant 1$ is in the domain of attraction of a non-degenerate stable distribution $G_{\beta}$ with index $1<\beta \leqslant 2$, i.e.,

$$
S_{n} / c_{n} \stackrel{d}{\rightarrow} G_{\beta}
$$

where $\left\{c_{n}\right\}$ is a non-decreasing positive sequence regular at $\infty$ with index $\beta^{-1}$. Then $\left\{S_{n}\right\}_{n} \geqslant 1$ is (Harris) recurrent with the counting measure $\pi(\cdot)$ on $\mathbf{Z}$ as its invariant measure. By (2.j) in Le Gall and Rosen [25],

$$
a(n) \equiv \sum_{k=1}^{n} P\left\{S_{k}=0\right\} \sim p(0) \sum_{k=1}^{n} \frac{1}{c_{k}} \quad(n \rightarrow \infty)
$$

where $p(x)$ is the density of $G_{\beta}$. In particular, $\left\{S_{n}\right\}_{n} \geqslant 1$ is $p$-regular with $p=1-\beta^{-1}$.

The local time $L_{n}^{x}(x \in \mathbf{Z}, n=1,2, \ldots)$ is defined as

$$
L_{n}^{x}=\#\left\{1 \leqslant k \leqslant n ; S_{k}=x\right\} .
$$

Let $x \in \mathbf{Z}$ be fixed. With $f(y)=1_{0}(y)-1_{x}(y)$ and $g(y)=1_{0}(y)$ we have

$$
\xi_{n}(t)=L_{[n t]}^{0}-L_{[n t]}^{x} \quad \text { and } \quad \eta_{n}(t)=L_{[n t]}^{0} \quad 0 \leqslant t \leqslant 1, n=1,2, \ldots
$$

The conditions in Theorem 1 are satisfied in this particular case. Notice that

$$
\begin{aligned}
\sigma_{f}^{2} & =\sum_{y \in \mathbf{Z}} \pi(y) f^{2}(y)+2 \sum_{k=1}^{\infty} \sum_{y \in \mathbf{Z}} \pi(y) f(y) P^{k} f(y) \\
& =2+4 \sum_{k=1}^{\infty}\left(P\left\{S_{k}=0\right\}-P\left\{S_{k}=x\right\}\right) \\
& =2+4 \sum_{k=1}^{\infty} \frac{1}{\pi} \int_{0}^{\pi}(1-\cos \lambda x) \varphi^{k}(\lambda) d \lambda \\
& =\frac{4}{\pi} \int_{0}^{\pi} \frac{1+\varphi(\lambda)}{1-\varphi(\lambda)}(1-\cos \lambda x) d \lambda
\end{aligned}
$$

and that

$$
\begin{aligned}
& \Lambda_{p}^{*}(x, y)= \begin{cases}\frac{x^{2}}{2 \sigma_{f}^{2} y}+\frac{1}{\beta}\left(\frac{\left(1-\beta^{-1}\right)^{1-\beta^{-1}} y}{\Gamma\left(2-\beta^{-1}\right)}\right)^{\beta} & y>0, \\
0 & \begin{array}{l}
x=y=0, \\
+\infty
\end{array}\end{cases} \\
& J_{p}(\gamma)= \begin{cases}\frac{1}{\beta} \int_{0}^{1}\left(\frac{\left(1-\beta^{-1}\right)^{1-\beta^{-1}} \dot{\gamma}(t)}{\Gamma\left(2-\beta^{-1}\right)}\right)^{\beta} d t & \text { if } \gamma \in \Gamma_{p}, \\
+\infty & \text { otherwise, }\end{cases} \\
& \kappa_{p}(\phi)= \begin{cases}\left(1+\frac{1}{\beta}\right) \int_{0}^{1}\left(\frac{\left(1-\beta^{-1}\right)^{1-\beta^{-1}} \dot{\phi}^{2}(t)}{2 \Gamma\left(2-\beta^{-1}\right) \sigma_{f}^{2}}\right)^{\frac{\beta}{1+\beta}} d t & \phi \in \Psi_{p}, \\
+\infty & \text { otherwise. }\end{cases}
\end{aligned}
$$


With the rate functions determined as the above, Theorems 1.1, 1.2 and 1.3 hold. In particular, taking the marginal distribution in Theorems 1.2 and 1.3 and by contraction principle we have

$$
\begin{aligned}
& \lim _{n \rightarrow \infty} \frac{1}{b_{n}} \log P\left\{L_{n}^{0} \geqslant \lambda a\left(n b_{n}^{-1}\right) b_{n}\right\}=-\frac{1}{\beta}\left(\frac{\left(1-\beta^{-1}\right)^{1-\beta^{-1}} \lambda}{\Gamma\left(2-\beta^{-1}\right)}\right)^{\beta}, \\
& \lim _{n \rightarrow \infty} \frac{1}{b_{n}} \log P\left\{L_{n}^{0}-L_{n}^{x} \geqslant \lambda \sqrt{a\left(n b_{n}^{-1}\right)} b_{n}\right\}=-\left(1+\frac{1}{\beta}\right)\left(\frac{\left(1-\beta^{-1}\right)^{1-\beta^{-1}} \lambda^{2}}{2 \Gamma\left(2-\beta^{-1}\right) \sigma_{f}^{2}}\right)^{\frac{\beta}{1+\beta}}
\end{aligned}
$$

for any $\lambda>0$. As $b_{n}=\log \log n$, such results are essentially needed for the laws of the iterated logarithm given in Theorem 1.2 in Marcus and Rosen [26].

Unfortunately, we are not able to establish the functional moderate deviations for 2-dimensional square integrable random walks or 1-dimensional random walks within the Cauchy domain as they turn out to be 0-regular, the case which is not covered here.

The paper will be organized as follows. In the next Section, we give some useful lemmas for the proof of Theorem 1.1 presented in Section 3. We then establish the functional law of the iterated logarithm in Section 4, and derive results for interesting functionals in the spirit of Strassen [32] in Section 5.

Our approach for moderate deviations consists of three steps: First, we establish our results under the assumption on existence of an atom. Second, we extend the established conclusion to strongly aperiodic Markov chains via the splitting technology developed by Nummelin [28,29] and by Athreya and Ney [4]. Finally, through a resolvent approximation argument we push from the case of strongly aperiodic Markov chain further to the full generality allowed in our theorems.

What makes our situation different from the limit laws established for the marginals $\Xi_{n}(1), \xi_{n}(1)$ and $\eta_{n}(1)$ is the complicated structure of our model. In fact, the trajectories $\Xi_{n}, \xi_{n}$ and $\eta_{n}$ are much more sensitive to time shifting than their marginals $\Xi_{n}(1), \xi_{n}(1)$ and $\eta_{n}(1)$, which makes Markov property harder to apply. To achieve our goals, we develop some estimates (Lemma 2.1 and (2) of Lemma 2.2) for the upper bounds with uniformity on the initial distributions. On the other hand, the evidence suggests that such uniformity does not hold for the lower bounds unless the Markov chain is uniformly recurrent. To establish the lower bounds, we develop a treatment for controlling the length of hitting time, which turns out to be helpful also in the proof of the functional LIL given in Theorem 4.1.

\section{Some lemmas}

Probably the most basic and important operation in Markovian systems is time shifting, which is closely associated with the Markov property. From now on, we use $\theta$ to denote the shifting operator of the Markov chain $\left\{X_{n}\right\}_{n} \geqslant 0$.

Lemma 2.1. Let $g \geqslant 0$ be special. Then for each $A \in \mathcal{E}^{+}$, there is an $\varepsilon>0$ such that

$$
\sup _{x \in E} E_{x} \exp \left\{\varepsilon \sum_{j=1}^{\tau_{A}} g\left(X_{j}\right)\right\}<+\infty,
$$

where the hitting time $\tau_{A}$ is defined as in (1.1).

Proof. The proof modifies some existing idea in literature (see, cf., Nummelin [29]). Without loss of generality, we assume that

$$
\sup _{x} g(x) \leqslant 1 .
$$


Define

$$
T_{m}=\inf \left\{n ; \sum_{j=1}^{n} g\left(X_{j}\right) \geqslant m\right\} m=1,2, \ldots
$$

By (1.3), there is a $m>1$ such that

$$
\sup _{x \in E} P_{x}\left\{\tau_{A} \geqslant T_{m-1}\right\}=\sup _{x \in E} P_{x}\left\{\sum_{j=1}^{\tau_{A}} g\left(X_{j}\right) \geqslant m-1\right\} \leqslant \frac{1}{2} .
$$

Note that for $N \geqslant 1$,

$$
T_{N m}+\tau_{A} \circ \theta^{T_{N m}}=\inf \left\{k>T_{N m} ; X_{k} \in A\right\}=\tau_{A}
$$

on the event $\left\{\tau_{A} \geqslant T_{N m}+1\right\}$. Thus,

$$
\begin{aligned}
P_{x}\left\{\tau_{A} \geqslant T_{(N+1) m}\right\} & =P_{x}\left\{\tau_{A} \geqslant T_{(N+1) m}, \tau_{A} \geqslant T_{N m}+1\right\} \\
& \leqslant P_{x}\left\{\tau_{A} \circ \theta^{T_{N m}} \geqslant T_{(N+1) m}-T_{N m}, \tau_{A} \geqslant T_{N m}\right\} .
\end{aligned}
$$

On the other hand, as $\sup g \leqslant 1$,

$$
\sum_{j=T_{N m}+1}^{T_{(N+1) m}} g\left(X_{j}\right) \geqslant(N+1) m-\sum_{j=1}^{T_{N m}} g\left(X_{j}\right) \geqslant(N+1) m-N m-1=m-1
$$

which implies that

$$
T_{N m}+T_{m-1} \circ \theta^{T_{N m}}=\inf \left\{k \geqslant T_{N m}+1 ; \sum_{j=T_{N m}+1}^{k} g\left(X_{j}\right) \geqslant m-1\right\} \leqslant T_{(N+1) m} .
$$

Hence, for each $x \in E$,

$$
\begin{aligned}
P_{x}\left\{\tau_{A} \geqslant T_{(N+1) m}\right\} & \leqslant P_{x}\left\{\tau_{A} \circ \theta^{T_{N m}} \geqslant T_{m-1} \circ \theta^{T_{N m}}, \tau_{A} \geqslant T_{N m}\right\} \leqslant P_{x}\left\{\tau_{A} \geqslant T_{N m}\right\} \cdot \sup _{y \in E} P_{y}\left\{\tau_{A} \geqslant T_{m-1}\right\} \\
& \leqslant \frac{1}{2} P_{x}\left\{\tau_{A} \geqslant T_{N m}\right\} .
\end{aligned}
$$

Hence we have

$$
\sup _{x \in E} P_{x}\left\{\tau_{A} \geqslant T_{(N+1) m}\right\} \leqslant \frac{1}{2^{N+1}}, \quad N=1,2, \ldots .
$$

Thus,

$$
\sup _{x \in E} P_{x}\left\{\sum_{j=1}^{\tau_{A}} g\left(X_{j}\right) \geqslant(N+1) m\right\} \leqslant \frac{1}{2^{N+1}}, \quad N=1,2, \ldots
$$

which leads to (2.1).

Recall (see, cf., Nummelin [29]) that a set $\alpha \in \mathcal{E}^{+}$is called an atom of $\left\{X_{n}\right\}_{n} \geqslant 0$ (or its transition $P$ ) if

$$
P(x, \cdot)=P(y, \cdot) \quad \forall x, y \in \alpha .
$$

In this paper, results are proved first under the assumption on existence of an atom $\alpha$. So we adopt the following notations through the rest of the paper. 
First, notice that $P_{x}=P_{y}$ for all $x, y \in \alpha$ on the $\sigma$-algebra generated by $\left\{X_{n}\right\}_{n} \geqslant 1$. So we denote the common value by $P_{\alpha}$. Notations like $P(\alpha, \cdot)$ and $E_{\alpha}$ are also used in an obvious way. Write

$$
\tau_{\alpha}(0)=0 \quad \text { and } \quad \tau_{\alpha}(k+1)=\inf \left\{n>\tau_{\alpha}(k): X_{n} \in \alpha\right\} \quad(k \geqslant 0) .
$$

Set $\tau_{\alpha}=\tau_{\alpha}(1)$. Because of Harris recurrence, $\tau_{\alpha}(k)<\infty$ a.s. for all $k$.

Define

$$
i_{\alpha}(n)=\sum_{k=1}^{n} I_{\left\{X_{k} \in \alpha\right\}}, \quad n=1,2, \ldots
$$

Lemma 2.2. Let $\alpha$ be an atom and $h: E \rightarrow \mathbf{R}$ be a measurable function. Write

$$
\begin{aligned}
& U_{0}=\sum_{j=1}^{\tau_{\alpha}} h\left(X_{j}\right) \quad \text { and } \quad V_{0}=\sum_{j=1}^{\tau_{\alpha}}\left|h\left(X_{j}\right)\right|, \\
& l=\log E_{\alpha}\left(\exp \left\{t U_{0}-s \tau_{\alpha}\right\} I_{\left\{V_{0} \leqslant a, \tau_{\alpha} \leqslant b\right\}}\right), \\
& L=\log E_{\alpha} \exp \left\{t U_{0}-s \min \left\{\tau_{\alpha}, \lambda\right\}\right\} .
\end{aligned}
$$

(1) For any integer $n \geqslant 1$ and real numbers $a>0, b>0, s>0$, and $t$,

$$
E_{x}\left[\exp \left\{t \sum_{k=1}^{n} h\left(X_{k}\right)-i_{\alpha}(n) l\right\} P_{X_{n}}\left\{V_{0} \leqslant a, \tau_{\alpha} \leqslant b\right\}\right] \geqslant \mathrm{e}^{-a|t|} \exp \{s n\} E_{x} \exp \left\{t U_{0}-s \tau_{\alpha}\right\} .
$$

(2) For each $n \geqslant 1, s>0$, $t$, and $\lambda>0$,

$$
E_{x} \exp \left\{t \sum_{k=1}^{n} h\left(X_{k}\right)-i_{\alpha}(n) L\right\} \leqslant c^{-1} \exp \{s(\lambda+n)\} \cdot E_{x} \exp \left\{t U_{0}\right\}
$$

where

$$
c=\inf _{x} E_{x} \exp \left\{t \sum_{j=1}^{\tau_{\alpha}} h\left(X_{j}\right)\right\} .
$$

Proof. Notice that under $P_{x}$, the 3-dimensional random variables

$$
\left(\sum_{j=\tau_{\alpha}(k)+1}^{\tau_{\alpha}(k+1)} h\left(X_{j}\right), \sum_{j=\tau_{\alpha}(k)+1}^{\tau_{\alpha}(k+1)}\left|h\left(X_{j}\right)\right|, \tau_{\alpha}(k+1)-\tau_{\alpha}(k)\right), \quad k=1,2, \ldots,
$$

form an i.i.d. sequence, to which the random variables

$$
i_{\alpha}(n)+1=\inf \left\{k \geqslant 1 ; \tau_{\alpha}(k)>n\right\}, \quad n=1,2, \ldots,
$$

are stopping times. One can see that with respect to the filtration

$$
\sigma\left\{X_{j} ; 0 \leqslant j \leqslant \tau_{\alpha}(k)\right\}, \quad k=1,2, \ldots,
$$

the sequence

$$
M_{k}=\exp \left\{t \sum_{j=1}^{\tau_{\alpha}(k)} h\left(X_{j}\right)-s \tau_{\alpha}(k)-(k-1) l\right\} \prod_{j=1}^{k-1} 1_{\left\{\sum_{i=\tau_{\alpha}(j)+1}^{\tau_{\alpha}(j+1)}\left|h\left(X_{i}\right)\right| \leqslant a, \tau_{\alpha}(j+1)-\tau_{\alpha}(j) \leqslant b\right\}}, \quad k=1,2, \ldots
$$


becomes a martingale, where we use the convention that

$$
\prod_{j=1}^{0} a_{j}=1
$$

for any sequence $\left\{a_{j}\right\}$ (so $M_{1}=\exp \left(t U_{0}-s \tau_{\alpha}\right)$ in our case). By the well known Doob's stopping rule,

$$
\begin{aligned}
& E_{x}\left[\exp \left\{t \sum_{j=1}^{\tau_{\alpha}\left(i_{\alpha}(n)+1\right)} h\left(X_{j}\right)-s \tau_{\alpha}\left(i_{\alpha}(n)+1\right)-i_{\alpha}(n) l\right\} \prod_{j=1}^{i_{\alpha}(n)} 1_{\left\{\sum_{i=\tau_{\alpha}(j)+1}^{\tau_{\alpha}(j+1)}\left|h\left(X_{i}\right)\right| \leqslant a, \tau_{\alpha}(j+1)-\tau_{\alpha}(j) \leqslant b\right\}}\right] \\
& =E_{x} M_{1} \\
& =E_{x} \exp \left\{t U_{0}-s \tau_{\alpha}\right\} .
\end{aligned}
$$

Notice that $\tau_{\alpha}\left(i_{\alpha}(n)\right) \leqslant n<\tau_{\alpha}\left(i_{\alpha}(n)+1\right)$. Hence

$$
\begin{aligned}
& E_{x}\left[\exp \left\{t \sum_{j=1}^{\tau_{\alpha}\left(i_{\alpha}(n)+1\right)} h\left(X_{j}\right)-i_{\alpha}(n) l\right\} 1_{\left\{\sum_{i=n+1}^{\tau_{\alpha}\left(i_{\alpha}(n)+1\right)}\left|h\left(X_{i}\right)\right| \leqslant a, \tau_{\alpha}\left(i_{\alpha}(n)+1\right)-n \leqslant b\right\}}\right] \\
& \geqslant \exp \{s n\} E_{x} \exp \left\{t U_{0}-s \tau_{\alpha}\right\} \text {. }
\end{aligned}
$$

By the fact

$$
\tau_{\alpha}\left(i_{\alpha}(n)+1\right)=\inf \left\{k>n ; X_{k} \in \alpha\right\}=n+\tau_{\alpha} \circ \theta^{n}
$$

and by Markov property, the left hand side is equal to

$$
\begin{gathered}
E_{x}\left[\exp \left\{t \sum_{j=1}^{n} h\left(X_{j}\right)-i_{\alpha}(n) l\right\} E_{X_{n}}\left(\exp \left\{t U_{0}\right\} ; V_{0} \leqslant a, \tau_{\alpha} \leqslant b\right)\right] \\
\leqslant \mathrm{e}^{a|t|} E_{x}\left[\exp \left\{t \sum_{j=0}^{n} h\left(X_{j}\right)-i_{\alpha}(n) l\right\} P_{X_{n}}\left\{V_{0} \leqslant a, \tau_{\alpha} \leqslant b\right\}\right] .
\end{gathered}
$$

Therefore, we get assertion (1) in Lemma 2.2.

Similarly, applying Doob's stopping rule to the martingale

$$
\exp \left\{t \sum_{j=1}^{\tau_{\alpha}(k)} h\left(X_{j}\right)-s \sum_{j=0}^{k-1} \min \left\{\tau_{\alpha}(j+1)-\tau_{\alpha}(j), \lambda\right\}-(k-1) L\right\}, \quad k=1,2, \ldots,
$$

gives

$$
\begin{aligned}
& E_{x} \exp \left\{t \sum_{j=1}^{\tau_{\alpha}\left(i_{\alpha}(n)+1\right)} h\left(X_{j}\right)-s \sum_{j=0}^{i_{\alpha}(n)} \min \left\{\tau_{\alpha}(j+1)-\tau_{\alpha}(j), \lambda\right\}-i_{\alpha}(n) L\right\} \\
& =E_{x} \exp \left\{t U_{0}-s \min \left(\tau_{\alpha}, \lambda\right)\right\} \\
& \leqslant E_{x} \exp \left\{t U_{0}\right\} .
\end{aligned}
$$

From the fact that

$$
\sum_{j=0}^{i_{\alpha}(n)} \min \left\{\tau_{\alpha}(j+1)-\tau_{\alpha}(j), \lambda\right\} \leqslant \tau_{\alpha}\left(i_{\alpha}(n)\right)+\lambda \leqslant n+\lambda .
$$


We have

$$
E_{x} \exp \left\{t \sum_{j=1}^{\tau_{\alpha}\left(i_{\alpha}(n)+1\right)} h\left(X_{j}\right)-i_{\alpha}(n) L\right\} \leqslant \mathrm{e}^{s(n+\lambda)} E_{x} \exp \left\{t U_{0}\right\}
$$

By Markov property, the left hand side becomes

$$
E_{x}\left[\exp \left\{t \sum_{j=1}^{n} h\left(X_{j}\right)-i_{\alpha}(n) L\right\} E_{X_{n}} \exp \left\{t U_{0}\right\}\right] \geqslant c E_{x} \exp \left\{t \sum_{j=1}^{n} h\left(X_{j}\right)-i_{\alpha}(n) L\right\} .
$$

Hence, we get assertion (2) of Lemma 2.2.

The next lemma presents sufficient conditions for LDP in $D[0,1]$, namely the finite dimensional LDP and exponential tightness in $D[0,1]$. We refer to Djellout, Guillin and $\mathrm{Wu}$ [16] for the proof.

Lemma 2.3. Let $\left(X^{n}(t)_{0 \leqslant t \leqslant 1}\right)_{n \geqslant 0}$ be a sequence of real right continuous left limit processes defined on $(\Omega, \mathcal{F}, P)$. Let $(\lambda(n))_{n \geqslant 0}$ be a sequence of positive numbers tending to infinity, and $D[0,1]$ be the space of real right continuous left limit functions equipped with the uniform convergence topology. Assume

(i) For every finite partition $\mathcal{P}=\left(t_{1}, \ldots, t_{m}\right)$ of $[0,1], P\left(\left(X^{n}\left(t_{1}\right), \ldots, X^{n}\left(t_{m}\right)\right) \in \cdot\right)$ satisfies the LDP on $R^{\mathcal{P}}$ with speed $\lambda(n)$ and with the rate function $I^{\mathcal{P}}$;

(ii) $\forall \delta>0$,

$$
\lim _{\varepsilon \rightarrow 0} \sup _{0 \leqslant s \leqslant 1} \limsup _{n \rightarrow \infty} \frac{1}{\lambda(n)} \log P\left(\sup _{s \leqslant t \leqslant s+\varepsilon}\left|X^{n}(t)-X^{n}(s)\right|>\delta\right)=-\infty
$$

(convention: $\left.\forall t>1, X^{n}(t):=X^{n}(1)\right)$. Then $P\left(X^{n} \in \cdot\right)$ satisfies a LDP on $D[0,1]$ w.r.t the uniform convergence topology with the same speed $\lambda(n)$ and with the rate function given by

$$
I(\gamma)=\sup _{\mathcal{P}} I^{\mathcal{P}}\left(\left(\gamma\left(t_{1}\right), \ldots, \gamma\left(t_{m}\right)\right)\right),
$$

where the supremum is taken over all finite partitions of $[0,1]$. Moreover $[I<+\infty]$ is a subset of the space $C[0,1]$.

\section{Proof of moderate deviations}

We only prove Theorem 1.1 , for Theorem 1.2 is a corollary of Theorem 1.1 and for the singularity brought by $\eta_{n}$ in the case $p=1$ will not affect $\xi_{n}$ and so we will have Theorem 1.3 if we take $g=0$ in the proof presented below.

The proof is carried out in three steps. In the first one, we prove the result under an atom assumption, then when the chain has some small set and then the general case.

Step 1. The atomic case.

In this step we prove the result under the extra assumption on the existence of an atom $\alpha \in \mathcal{E}^{+}$. We will carry out here the plan of the Lemma 2.3, first establishing the finite dimensional moderate deviation principle and then the exponential continuity (ii).

a) We first assume that $g=I_{\alpha}$. We show that for any finite partition $\mathcal{P}$ on $[0,1]$ : $0=t_{0}<t_{1}<t_{2}<\cdots<t_{m}=1$, and any real numbers $A_{1}, \ldots, A_{m} ; B_{1}, \ldots, B_{m}$,

$$
\begin{aligned}
& \lim _{n \rightarrow \infty} \frac{1}{b_{n}} \log E_{\mu} \exp \left\{\frac{1}{\sqrt{a\left(n b_{n}^{-1}\right)}} \sum_{k=1}^{m} A_{k}\left(\xi_{n}\left(t_{k}\right)-\xi_{n}\left(t_{k-1}\right)\right)+\frac{1}{a\left(n b_{n}^{-1}\right)} \sum_{k=1}^{m} B_{k}\left(\eta_{n}\left(t_{k}\right)-\eta_{n}\left(t_{k-1}\right)\right)\right\} \\
& =\sum_{k=1}^{m}\left(t_{k}-t_{k-1}\right) \Lambda_{p}\left(A_{k}, B_{k}\right),
\end{aligned}
$$


where

$$
\Lambda_{p}(a, b)= \begin{cases}{\left[\Gamma(p+1)\left(\frac{a^{2} \sigma_{f}^{2}}{2}+b \pi(\alpha)\right)\right]^{1 / p}} & \frac{a^{2} \sigma_{f}^{2}}{2}+b \pi(\alpha) \geqslant 0 \\ 0 & \frac{a^{2} \sigma_{f}^{2}}{2}+b \pi(\alpha)<0\end{cases}
$$

Let $n_{k}=\left[n t_{k}\right](k=1,2, \ldots, m)$. By the estimates

$$
\begin{aligned}
& \left|\left(\xi_{n}\left(t_{k}\right)-\xi_{n}\left(t_{k-1}\right)\right)-\left(\xi_{n_{k}}(1)-\xi_{n_{k-1}}(1)\right)\right| \leqslant 2\|f\|_{\infty}, \\
& \left|\left(\eta_{n}\left(t_{k}\right)-\eta_{n}\left(t_{k-1}\right)\right)-\left(i_{\alpha}\left(n_{k}\right)-i_{\alpha}\left(n_{k-1}\right)\right)\right| \leqslant 2\|g\|_{\infty},
\end{aligned}
$$

we only need to show

$$
\begin{aligned}
& \lim _{n \rightarrow \infty} \frac{1}{b_{n}} \log E_{\mu} \exp \left\{\frac{1}{\sqrt{a\left(n b_{n}^{-1}\right)}} \sum_{k=1}^{m} A_{k}\left(\xi_{n_{k}}(1)-\xi_{n_{k-1}}(1)\right)+\frac{1}{a\left(n b_{n}^{-1}\right)} \sum_{k=1}^{m} B_{k}\left(i_{\alpha}\left(n_{k}\right)-i_{\alpha}\left(n_{k-1}\right)\right)\right\} \\
& =\sum_{k=1}^{m}\left(t_{k}-t_{k-1}\right) \Lambda_{p}\left(A_{k}, B_{k}\right) .
\end{aligned}
$$

By Lemma 2.3 of Chen [9], by Lemma 2.1 (which enables us to give up the imposed boundedness in Chen [9]) and by the fact (see p. 120 in Chen [9]) that $E_{\alpha} U^{2}=\pi(\alpha)^{-1} \sigma_{f}^{2}$, we have that for each $1 \leqslant k \leqslant m$ and $s \geqslant 0$

$$
\log E_{\alpha} \exp \left\{\frac{A_{k}}{\sqrt{a\left(n b_{n}^{-1}\right)}} \sum_{k=1}^{\tau_{\alpha}} f\left(X_{k}\right)-\frac{s b_{n}}{n} \tau_{\alpha}\right\} \sim \frac{1}{\pi(\alpha) \cdot a\left(n b_{n}^{-1}\right)}\left(\frac{A_{k}^{2} \sigma_{f}^{2}}{2}-\frac{s^{p}}{\Gamma(p+1)}\right) \quad(n \rightarrow \infty) .
$$

Let

$$
U=\sum_{j=1}^{\tau_{\alpha}} f\left(X_{j}\right) \quad \text { and } \quad V=\sum_{j=1}^{\tau_{\alpha}}\left|f\left(X_{j}\right)\right| .
$$

We therefore have,

$$
\begin{aligned}
l_{n, k}(s) & \equiv \log E_{\alpha}\left[\exp \left\{\frac{A_{k}}{\sqrt{a\left(n b_{n}^{-1}\right)}} \sum_{k=1}^{\tau_{\alpha}} f\left(X_{k}\right)-\frac{s b_{n}}{n} \tau_{\alpha}\right\} ; V \leqslant \varepsilon \sqrt{a\left(n b_{n}^{-1}\right)} b_{n}, \tau_{\alpha} \leqslant \varepsilon n\right] \\
& \sim \frac{1}{\pi(\alpha) \cdot a\left(n b_{n}^{-1}\right)}\left(\frac{A_{k}^{2} \sigma_{f}^{2}}{2}-\frac{s^{p}}{\Gamma(p+1)}\right) \quad(n \rightarrow \infty), \\
L_{n, k}(s) & \equiv \log E_{\alpha} \exp \left\{\frac{A_{k}}{\sqrt{a\left(n b_{n}^{-1}\right)}} \sum_{k=1}^{\tau_{\alpha}} f\left(X_{k}\right)-\frac{s b_{n}}{n} \min \left\{\tau_{\alpha}, \varepsilon n\right\}\right\} \\
& \sim \frac{1}{\pi(\alpha) \cdot a\left(n b_{n}^{-1}\right)}\left(\frac{A_{k}^{2} \sigma_{f}^{2}}{2}-\frac{s^{p}}{\Gamma(p+1)}\right) \quad(n \rightarrow \infty) .
\end{aligned}
$$

We will divide the proof of (3.2) in two parts: the upper bound and then the more difficult lower bound. Upper bound of (3.2).

To establish this upper bound, notice that by Markov property 


$$
\begin{aligned}
& E_{\mu} \exp \left\{\frac{1}{\sqrt{a\left(n b_{n}^{-1}\right)}} \sum_{k=1}^{m} A_{k}\left(\xi_{n_{k}}(1)-\xi_{n_{k-1}}(1)\right)+\frac{1}{a\left(n b_{n}^{-1}\right)} \sum_{k=1}^{m} B_{k}\left(i_{\alpha}\left(n_{k}\right)-i_{\alpha}\left(n_{k-1}\right)\right)\right\} \\
& \leqslant \prod_{k=1}^{m} \sup _{x} E_{x} \exp \left\{\frac{A_{k}}{\sqrt{a\left(n b_{n}^{-1}\right)}} \xi_{n_{k}-n_{k-1}}(1)+\frac{B_{k}}{a\left(n b_{n}^{-1}\right)} i_{\alpha}\left(n_{k}-n_{k-1}\right)\right\} .
\end{aligned}
$$

For any $1 \leqslant k \leqslant m, \varepsilon>0$ and $s>\Lambda_{p}\left(A_{k}, B_{k}\right)$, by (3.4),

$$
L_{n, k}(s)<-\frac{B_{k}}{a\left(n b_{n}^{-1}\right)}
$$

as $n$ is sufficiently large. Therefore, by Lemma 2.2(2)

$$
\begin{aligned}
& \sup _{x} E_{x} \exp \left\{\frac{A_{k}}{\sqrt{a\left(n b_{n}^{-1}\right)}} \xi_{n_{k}-n_{k-1}}(1)+\frac{B_{k}}{a\left(n b_{n}^{-1}\right)} i_{\alpha}\left(n_{k}-n_{k-1}\right)\right\} \\
& \quad \leqslant \sup _{x} E_{x} \exp \left\{\frac{A_{k}}{\sqrt{a\left(n b_{n}^{-1}\right)}} \xi_{n_{k}-n_{k-1}}(1)-i_{\alpha}\left(n_{k}-n_{k-1}\right) L_{n, k}(s)\right\} \\
& \leqslant c_{n}^{-1} \exp \left\{\left(\varepsilon n+n_{k}-n_{k-1}\right) \cdot \frac{s b_{n}}{n}\right\} \sup _{x} E_{x} \exp \left\{\frac{A_{k}}{\sqrt{a\left(n b_{n}^{-1}\right)}} U\right\} \\
& \sim \exp \left\{\left(\varepsilon n+n_{k}-n_{k-1}\right) \cdot \frac{s b_{n}}{n}\right\} \quad(n \rightarrow \infty),
\end{aligned}
$$

where

$$
c_{n}=\inf _{x} E_{x} \exp \left\{\frac{A_{k}}{\sqrt{a\left(n b_{n}^{-1}\right)}} U\right\}
$$

and the last step follows from Lemma 2.1. Consequently, for each $1 \leqslant k \leqslant m$,

$$
\begin{aligned}
& \limsup _{n \rightarrow \infty} \frac{1}{b_{n}} \log \sup _{x} E_{x} \exp \left\{\frac{A_{k}}{\sqrt{a\left(n b_{n}^{-1}\right)}} \xi_{n_{k}-n_{k-1}}(1)+\frac{B_{k}}{a\left(n b_{n}^{-1}\right)} i_{\alpha}\left(n_{k}-n_{k-1}\right)\right\} \\
& \leqslant\left(t_{k}-t_{k-1}\right) \Lambda_{p}\left(A_{k}, B_{k}\right) .
\end{aligned}
$$

Summarizing what we have discussed, we obtain the desired upper bound:

$$
\begin{aligned}
& \limsup _{n \rightarrow \infty} \frac{1}{b_{n}} \log E_{\mu} \exp \left\{\frac{1}{\sqrt{a\left(n b_{n}^{-1}\right)}} \sum_{k=1}^{m} A_{k}\left(\xi_{n_{k}}(1)-\xi_{n_{k-1}}(1)\right)+\frac{1}{a\left(n b_{n}^{-1}\right)} \sum_{k=1}^{m} B_{k}\left(i_{\alpha}\left(n_{k}\right)-i_{\alpha}\left(n_{k-1}\right)\right)\right\} \\
& \leqslant \sum_{k=1}^{m}\left(t_{k}-t_{k-1}\right) \Lambda_{p}\left(A_{k}, B_{k}\right) .
\end{aligned}
$$

We may now pass to the proof of the lower bound.

Lower bound of (3.2).

The proof of the lower bound for (3.2) is more delicate. Let

$$
\Delta_{k}=\frac{A_{k}}{\sqrt{a\left(n b_{n}^{-1}\right)}}\left(\xi_{n_{k}}(1)-\xi_{n_{k-1}}(1)\right)+\frac{B_{k}}{a\left(n b_{n}^{-1}\right)}\left(i_{\alpha}\left(n_{k}\right)-i_{\alpha}\left(n_{k-1}\right)\right)
$$




$$
=\left(\frac{A_{k}}{\sqrt{a\left(n b_{n}^{-1}\right)}} \xi_{n_{k}-n_{k-1}}(1)+\frac{B_{k}}{a\left(n b_{n}^{-1}\right)} i_{\alpha}\left(n_{k}-n_{k-1}\right)\right) \circ \theta^{n_{k-1}}=W_{k} \circ \theta^{n_{k-1}} .
$$

Then for any $\varepsilon>0$, by Markov property, and recall $n_{m}=n$,

$$
\begin{aligned}
I_{m} & \equiv E_{\mu}\left(\exp \left\{\sum_{k=1}^{m} \Delta_{k}\right\} ; V \circ \theta^{n} \leqslant \varepsilon \sqrt{a\left(n b_{n}^{-1}\right)} b_{n}, \tau_{\alpha} \circ \theta^{n} \leqslant \varepsilon n\right) \\
& =E_{\mu}\left(\exp \left\{\sum_{k=1}^{m} \Delta_{k}\right\} P_{X_{n_{m}}}\left[V \leqslant \varepsilon \sqrt{a\left(n b_{n}^{-1}\right)} b_{n}, \tau_{\alpha} \leqslant \varepsilon n\right]\right) \\
& =E_{\mu}\left(\exp \left\{\sum_{k=1}^{m-1} \Delta_{k}\right\} E_{X_{n_{m-1}}}\left(\exp \left\{W_{m}\right\} P_{X_{n_{m}-n_{m-1}}}\left[V \leqslant \varepsilon \sqrt{a\left(n b_{n}^{-1}\right)} b_{n}, \tau_{\alpha} \leqslant \varepsilon n\right]\right)\right) .
\end{aligned}
$$

Let $1 \leqslant k \leqslant m, \varepsilon>0$. Let us first assume that $\Lambda_{p}\left(A_{k}, B_{k}\right)>0$ and take then $0<s_{k}<\Lambda_{p}\left(A_{k}, B_{k}\right)$ and by (3.3) we have

$$
l_{n, k}\left(s_{k}\right)>-\frac{B_{k}}{a\left(n b_{n}^{-1}\right)}
$$

for sufficiently large $n$.

As $\Lambda_{p}\left(A_{m}, B_{m}\right)>0$, from (1) in Lemma 2.2, we have that for any $x \in E$,

$$
\begin{aligned}
E_{x} & \left(\exp \left\{W_{m}\right\} P_{X_{n_{m}-n_{m-1}}}\left[V \leqslant \varepsilon \sqrt{a\left(n b_{n}^{-1}\right)} b_{n}, \tau_{\alpha} \leqslant \varepsilon n\right]\right) \\
\geqslant & E_{x}\left(\exp \left\{\frac{A_{m}}{\sqrt{a\left(n b_{n}^{-1}\right)}} \xi_{n_{m}-n_{m-1}}(1)-l_{n, m}\left(s_{m}\right) i_{\alpha}\left(n_{m}-n_{m-1}\right)\right\}\right. \\
& \left.\times P_{X_{n_{m}-n_{m-1}}}\left[V \leqslant \varepsilon \sqrt{a\left(n b_{n}^{-1}\right)} b_{n}, \tau_{\alpha} \leqslant \varepsilon n\right]\right) \\
\geqslant & \mathrm{e}^{-\varepsilon b_{n}} E_{x} \exp \left\{\frac{A_{m}}{\sqrt{a\left(n b_{n}^{-1}\right)}} \sum_{j=1}^{\tau_{\alpha}} f\left(X_{j}\right)-\frac{s_{m} b_{n}}{n} \tau_{\alpha}\right\} \exp \left\{\left(n_{m}-n_{m-1}\right) \cdot \frac{s_{m} b_{n}}{n}\right\} \\
\geqslant & \exp \left\{-\left(1+\left|A_{m}\right|+s_{m}\right) \varepsilon b_{n}\right\} \exp \left\{\left(n_{m}-n_{m-1}\right) \cdot \frac{s_{m} b_{n}}{n}\right\} P_{x}\left\{V \leqslant \varepsilon \sqrt{a\left(n b_{n}^{-1}\right)} b_{n}, \tau_{\alpha} \leqslant \varepsilon n\right\} .
\end{aligned}
$$

We now establish the inequality (3.7) in the case $\Lambda_{p}\left(A_{m}, B_{m}\right)=0$ for large $n$ (with $s_{m}=\delta$ for any fixed but arbitrary $\delta>0)$. Indeed, by Jensen inequality

$$
\begin{aligned}
& E_{x}\left(\exp \left\{W_{m}\right\} P_{X_{n_{m}-n_{m-1}}}\left[V \leqslant \varepsilon \sqrt{a\left(n b_{n}^{-1}\right)} b_{n}, \tau_{\alpha} \leqslant \varepsilon n\right]\right) \\
& \geqslant \exp \left\{\frac{A_{m}}{\sqrt{a\left(n b_{n}^{-1}\right)}} E_{x} \xi_{n_{m}-n_{m-1}}(1)+\frac{B_{m}}{a\left(n b_{n}^{-1}\right)} E_{x} i_{\alpha}\left(n_{m}-n_{m-1}\right)\right\} \\
& \quad \times E_{x}\left(P_{X_{n_{m}-n_{m-1}}}\left[V \leqslant \varepsilon \sqrt{a\left(n b_{n}^{-1}\right)} b_{n}, \tau_{\alpha} \leqslant \varepsilon n\right]\right) .
\end{aligned}
$$

Hence, our claim follows from the following observations (i), (ii) and (iii): 
(i). Taking $A_{m}=B_{m}=0$ and applying (1) in Lemma 2.2 give that

$$
\begin{aligned}
& E_{x}\left(P_{X_{n_{m}-n_{m-1}}}\left[V \leqslant \varepsilon \sqrt{a\left(n b_{n}^{-1}\right)} b_{n}, \tau_{\alpha} \leqslant \varepsilon n\right]\right) \\
& \geqslant \exp \left\{-\varepsilon b_{n}\right\} P_{x}\left\{V \leqslant \varepsilon \sqrt{a\left(n b_{n}^{-1}\right)} b_{n}, \tau_{\alpha} \leqslant \varepsilon n\right\} .
\end{aligned}
$$

(ii). The sequence $\left\{\sup _{x \in E}\left|E_{x} \xi_{n}(1)\right|\right\}_{n \geqslant 1}$ is bounded (Proposition 5.7, Chapter 6 of Revuz [33]), so that

$$
\exp \left\{\frac{A_{m}}{\sqrt{a\left(n b_{n}^{-1}\right)}} E_{x} \xi_{n_{m}-n_{m-1}}(1)\right\}
$$

is arbitrarily close to 1 for large $n$ as $a\left(n / b_{n}\right) \rightarrow \infty$.

(iii). By the estimate,

$$
E_{x} i_{\alpha}(n)=E_{x}\left(i_{\alpha}(n) I_{\left\{\tau_{\alpha} \leqslant n\right\}}\right)=\sum_{k=1}^{n} E_{x}\left(1+E_{x} \sum_{j=k+1}^{n} I_{\left\{X_{j} \in \alpha\right\}}\right) I_{\left\{\tau_{\alpha}=k\right\}} \leqslant 1+E_{\alpha} i_{\alpha}(n)
$$

we have

$$
\sup _{x \in E} E_{x} i_{\alpha}(n) \sim a(n) \quad(n \rightarrow \infty),
$$

see Proposition 5.13(iii) in Nummelin [29]. It enables us to get that for sufficiently large $n$,

$$
\exp \left\{\frac{B_{m}}{a\left(n b_{n}^{-1}\right)} E_{x} i_{\alpha}\left(n_{m}-n_{m-1}\right)\right\} \geqslant \exp \left\{-\frac{\left|B_{m}\right| a(n)}{a\left(n b_{n}^{-1}\right)}\right\} \sim \exp \left\{-\left|B_{m}\right| b_{n}^{p}\right\} \geqslant \mathrm{e}^{-\delta \varepsilon b_{n}}
$$

for some arbitrary $\delta$, which enables us to prove (3.7) in the case $\Lambda_{p}\left(A_{m}, B_{m}\right)=0$ for large $n$.

Therefore, returning to the proof of our lower bound,

$$
\begin{aligned}
I_{m} \geqslant & \exp \left\{-\left(1+\left|A_{m}\right|+s_{m}\right) \varepsilon b_{n}\right\} \exp \left\{\left(n_{m}-n_{m-1}\right) \cdot \frac{s_{m} b_{n}}{n}\right\} \\
& \times E_{\mu}\left(\exp \left\{\sum_{k=1}^{m-1} \Delta_{k}\right\} P_{X_{n_{m-1}}}\left\{V \leqslant \varepsilon \sqrt{a\left(n b_{n}^{-1}\right)} b_{n}, \tau_{\alpha} \leqslant \varepsilon n\right\}\right) \\
= & \exp \left\{-\left(1+\left|A_{m}\right|+s_{m}\right) \varepsilon b_{n}\right\} \exp \left\{\left(n_{m}-n_{m-1}\right) \cdot \frac{s_{m} b_{n}}{n}\right\} I_{m-1} .
\end{aligned}
$$

Repeating the procedure we have

$$
\begin{aligned}
I_{m} \geqslant & P_{\mu}\left\{V \leqslant \varepsilon \sqrt{a\left(n b_{n}^{-1}\right)} b_{n}, \tau_{\alpha} \leqslant \varepsilon n\right\} \\
& \times \exp \left\{-\varepsilon b_{n} \sum_{k=1}^{m}\left(1+\left|A_{k}\right|+s_{k}\right)\right\} \exp \left\{\frac{b_{n}}{n} \sum_{k=1}^{m}\left(n_{k}-n_{k-1}\right) s_{k}\right\}
\end{aligned}
$$

for sufficiently large $n$. Since $s_{k}$ can be arbitrarily close to $\Lambda_{p}\left(A_{k}, B_{k}\right)$ for each $1 \leqslant k \leqslant m$

$$
\liminf _{n \rightarrow \infty} \frac{1}{b_{n}} \log I_{m} \geqslant-\varepsilon \sum_{k=1}^{m}\left(1+\left|A_{k}\right|+\Lambda_{p}\left(A_{k}, B_{k}\right)\right)+\sum_{k=1}^{m}\left(t_{k}-t_{k-1}\right) \Lambda_{p}\left(A_{k}, B_{k}\right) .
$$

Notice that the left hand side is decreasing in $\varepsilon$, while the right hand side is increasing in $\varepsilon$. We must have, for each $\varepsilon>0$,

$$
\liminf _{n \rightarrow \infty} \frac{1}{b_{n}} \log I_{m} \geqslant \sum_{k=1}^{m}\left(t_{k}-t_{k-1}\right) \Lambda_{p}\left(A_{k}, B_{k}\right)
$$


Consequently, we obtain the lower bound

$$
\begin{aligned}
& \liminf _{n \rightarrow \infty} \frac{1}{b_{n}} \log E_{\mu} \exp \left\{\frac{1}{\sqrt{a\left(n b_{n}^{-1}\right)}} \sum_{k=1}^{m} A_{k}\left(\xi_{n_{k}}(1)-\xi_{n_{k-1}}(1)\right)+\frac{1}{a\left(n b_{n}^{-1}\right)} \sum_{k=1}^{m} B_{k}\left(\eta_{n_{k}}(1)-\eta_{n_{k-1}}(1)\right)\right\} \\
& \geqslant \sum_{k=1}^{m}\left(t_{k}-t_{k-1}\right) \Lambda_{p}\left(A_{k}, B_{k}\right) .
\end{aligned}
$$

Therefore, (3.2) follows from (3.6) and (3.9).

b) Remark now that by (3.1) and Gärtner-Ellis Theorem (see Dembo and Zeitouni [15] and 2.3) we have the moderate deviations for

$$
\left(\left\{\frac{1}{\sqrt{a\left(n b_{n}^{-1}\right) b_{n}}}\left(\xi_{n}\left(t_{k}\right)-\xi_{n}\left(t_{k-1}\right)\right)\right\}_{1 \leqslant k \leqslant m},\left\{\frac{1}{a\left(n b_{n}^{-1}\right) b_{n}}\left(\eta_{n}\left(t_{k}\right)-\eta_{n}\left(t_{k-1}\right)\right)\right\}_{1 \leqslant k \leqslant m}\right)
$$

with rate function

$$
\Lambda_{m}^{*}\left(\left(x_{i}\right),\left(y_{i}\right)\right)=\sum_{k=1}^{m}\left(t_{k}-t_{k-1}\right) \Lambda_{p}^{*}\left(\frac{x_{k}}{t_{k}-t_{k-1}}, \frac{y_{k}}{t_{k}-t_{k-1}}\right) .
$$

Indeed, the rate function is defined by

$$
\begin{aligned}
& \Lambda_{m}^{*}\left(\left(x_{1}, \ldots, x_{m}\right),\left(y_{1}, \ldots, y_{m}\right)\right) \\
& =\sup _{A, B \in R^{m}}\left\{\langle A, x\rangle+\langle B, y\rangle-\sum_{k=1}^{m}\left(t_{k}-t_{k-1}\right) \Lambda_{p}\left(A_{k}, B_{k}\right)\right\} \\
& =\sup _{A, B \in R^{m}}\left\{\sum_{k=1}^{m}\left(A_{k} x_{k}+B_{k} y_{k}-\left(t_{k}-t_{k-1}\right) \Lambda_{p}\left(A_{k}, B_{k}\right)\right)\right\} \\
& =\sum_{k=1}^{m}\left(t_{k}-t_{k-1}\right) \sup _{A, B \in R}\left\{\frac{A x_{k}}{t_{k}-t_{k-1}}+\frac{B y_{k}}{t_{k}-t_{k-1}}-\Lambda_{p}(A, B)\right\}
\end{aligned}
$$

which gives the result by a direct computation of the Legendre transform of $\Lambda_{p}$. We conclude by the contraction principle (see Theorem 4.2.1 in Dembo and Zeitouni [15]) to deduce the moderate deviations of

$$
\left(\left\{\frac{1}{\sqrt{a\left(n b_{n}^{-1}\right) b_{n}}} \xi_{n}\left(t_{k}\right)\right\}_{1 \leqslant k \leqslant m},\left\{\frac{1}{a\left(n b_{n}^{-1}\right) b_{n}} \eta_{n}\left(t_{k}\right)\right\}_{1 \leqslant k \leqslant m}\right)
$$

with rate function

$$
I_{p}^{\mathcal{P}}(x, y)=\sum_{k=1}^{m}\left(t_{k}-t_{k-1}\right) \Lambda_{p}^{*}\left(\frac{x_{k}-x_{k-1}}{t_{k}-t_{k-1}}, \frac{y_{k}-y_{k-1}}{t_{k}-t_{k-1}}\right) .
$$

We have so shown the finite dimensional moderate deviations which are exactly condition (i) of Lemma 2.3.

c) We now claim that for any $\delta>0$

$$
\begin{aligned}
& \lim _{\varepsilon \rightarrow 0} \sup _{s \in[0,1]} \limsup _{n \rightarrow \infty} \frac{1}{b_{n}} \log P_{\mu}\left\{\sup _{s \leqslant t \leqslant s+\varepsilon}\left|\xi_{n}(t)-\xi_{n}(s)\right|>\delta \sqrt{a\left(n b_{n}^{-1}\right)} b_{n}\right\}=-\infty \\
& \lim _{\varepsilon \rightarrow 0} \sup _{s \in[0,1]} \limsup _{n \rightarrow \infty} \frac{1}{b_{n}} \log P_{\mu}\left\{\sup _{s \leqslant t \leqslant s+\varepsilon}\left|\eta_{n}(t)-\eta_{n}(s)\right|>\delta a\left(n b_{n}^{-1}\right) b_{n}\right\}=-\infty .
\end{aligned}
$$


We only prove (3.10), as the proof of (3.11) is analogous (and even simpler due to the monotonicity of $\eta_{n}(\cdot)$ ). Notice that (3.10) is equivalent to

$$
\lim _{\varepsilon \rightarrow 0} \limsup _{n \rightarrow \infty} \frac{1}{b_{n}} \log \max _{k \leqslant n-\varepsilon n} P_{\mu}\left\{\max _{l \leqslant \varepsilon n}\left|\sum_{j=k+1}^{k+l} f\left(X_{j}\right)\right|>\delta \sqrt{a\left(n b_{n}^{-1}\right)} b_{n}\right\}=-\infty .
$$

By Markov property

$$
P_{\mu}\left\{\max _{l \leqslant \varepsilon n}\left|\sum_{j=k+1}^{k+l} f\left(X_{j}\right)\right|>\delta \sqrt{a\left(n b_{n}^{-1}\right)} b_{n}\right\} \leqslant \sup _{x \in E} P_{x}\left\{\max _{l \leqslant \varepsilon n}\left|\sum_{j=1}^{l} f\left(X_{j}\right)\right|>\delta \sqrt{a\left(n b_{n}^{-1}\right)} b_{n}\right\} .
$$

Notice that

$$
\max _{l \leqslant \varepsilon n}\left|\sum_{j=1}^{l} f\left(X_{j}\right)\right| \leqslant \max _{1 \leqslant k \leqslant i_{\alpha}([\varepsilon n])}\left|\sum_{j=1}^{\tau_{\alpha}(k)} f\left(X_{j}\right)\right|+\max _{0 \leqslant k \leqslant i_{\alpha}([\varepsilon n])} \sum_{j=\tau_{\alpha}(k)+1}^{\tau_{\alpha}(k+1)}\left|f\left(X_{j}\right)\right| .
$$

Hence

$$
\begin{aligned}
& \sup _{x \in E} P_{x}\left\{\max _{l \leqslant \varepsilon n}\left|\sum_{j=1}^{l} f\left(X_{j}\right)\right|>\delta \sqrt{a\left(n b_{n}^{-1}\right)} b_{n}\right\} \\
& \leqslant \sup _{x \in E} P_{x}\left\{\max _{1 \leqslant k \leqslant i_{\alpha}([\varepsilon n])}\left|\sum_{j=1}^{\tau_{\alpha}(k)} f\left(X_{j}\right)\right|>2^{-1} \delta \sqrt{a\left(n b_{n}^{-1}\right)} b_{n}\right\} \\
& \quad+a\left(\frac{n}{b_{n}}\right) b_{n} \sup _{x \in E} P_{x}\left\{\sum_{j=1}^{\tau_{\alpha}}\left|f\left(X_{j}\right)\right|>2^{-1} \delta \sqrt{a\left(n b_{n}^{-1}\right)} b_{n}\right\}+\sup _{x \in E} P_{x}\left\{i_{\alpha}([\varepsilon n]) \geqslant a\left(n b_{n}^{-1}\right) b_{n}\right\} \\
& =(I)+(I I)+(I I I) . \quad \text { (say) }
\end{aligned}
$$

In the light of Lemma 2.1 we have that

$$
\limsup _{n \rightarrow \infty} \frac{1}{b_{n}} \log (I I)=-\infty \text {. }
$$

Notice that the estimate made in (3.5) actually gives

$$
\begin{aligned}
& \lim _{\varepsilon \rightarrow \infty} \limsup _{n \rightarrow \infty} \frac{1}{b_{n}} \log \sup _{x} E_{x} \exp \left\{\frac{\lambda}{\sqrt{a\left(n b_{n}^{-1}\right)}} \xi_{[\varepsilon n]}(1)\right\}=0, \quad \forall \lambda \in \mathbf{R}, \\
& \lim _{\varepsilon \rightarrow \infty} \limsup _{n \rightarrow \infty} \frac{1}{b_{n}} \log \sup _{x} E_{x} \exp \left\{\frac{\lambda}{a\left(n b_{n}^{-1}\right)} i_{\alpha}([\varepsilon n])\right\}=0, \quad \forall \lambda>0
\end{aligned}
$$

(this is where we need $p>0$ !). By Chebyshev inequality and (3.14) we obtain

$$
\lim _{\varepsilon \rightarrow 0} \limsup _{n \rightarrow \infty} \frac{1}{b_{n}} \log (I I I)=-\infty .
$$

By the maximal inequality given in Corollary 3.3, Chapter I of Chen [6],

$$
(I) \leqslant\left(1-u_{n}\right)^{-1} \sup _{x \in E} P_{x}\left\{\left|\xi_{[\varepsilon n]}(1)\right|>4^{-1} \delta \sqrt{a\left(n b_{n}^{-1}\right)} b_{n}\right\}
$$

where the sequence 


$$
\begin{aligned}
u_{n} & \equiv \max _{1 \leqslant k \leqslant \varepsilon n} P_{\alpha}\left\{\left|\xi_{k}(1)\right| \geqslant 4^{-1} \delta \sqrt{a\left(n b_{n}^{-1}\right)} b_{n}\right\} \quad n \geqslant 1 \\
& =\max _{1 \leqslant k \leqslant \varepsilon n} P_{\alpha}\left(\left|\frac{\xi_{k}(1)}{\sqrt{a(n)}}\right| \geqslant 4^{-1} \delta \sqrt{\frac{a\left(n b_{n}^{-1}\right)}{a(n)}} b_{n}\right)
\end{aligned}
$$

approaches 0 when $n \rightarrow \infty$, as a consequence of the central limit theorem given in Theorem 1.5, Chen [9] and the $p$-regularity of our chain. Therefore, by (3.13) and Chebyshev inequality we have

$$
\lim _{\varepsilon \rightarrow 0} \limsup _{n \rightarrow \infty} \frac{1}{b_{n}} \log (I)=-\infty \text {. }
$$

Summarizing what we have, we obtain (3.12) (and therefore (3.10)). Consequently condition (ii) of Lemma 2.3 is verified and we thus get the moderate deviations of $\left(\Xi_{n}(\cdot)\right)$ with rate function $\sup _{\mathcal{P}} I_{p}^{\mathcal{P}}$ which is easily shown to be equal to $I_{p}$ (by the convexity of $\Lambda_{p}^{*}$ and proof of Lemma 5.1.6 in Dembo and Zeitouni [15]). Hence, we have proved Theorem 1.1 in the case $g=I_{\alpha}$.

d) By the continuous map $(\phi(\cdot), \gamma(\cdot)) \mapsto(\phi(\cdot), \lambda \gamma(\cdot))$ and contraction principle, one can extend the achieved conclusion to the case when $g=\lambda I_{\alpha}$ for any constant $\lambda \geqslant 0$. For any given special function $g$, notice that the function $g-\pi(g) \pi(\alpha)^{-1} I_{\alpha}$ is a charge. Applying the upper bound of the moderate deviation we have proved to the sequence $\xi_{n}(\cdot)$ with $f$ being defined as $g-\pi(g) \pi(\alpha)^{-1} I_{\alpha}$, we have

$$
\begin{gathered}
\limsup _{n \rightarrow \infty} \frac{1}{b_{n}} \log P\left\{\max _{0 \leqslant t \leqslant 1}\left|\eta_{n}(t)-\zeta_{n}(t)\right| \geqslant r \sqrt{a\left(\frac{n}{b_{n}}\right)} b_{n}\right\} \\
\leqslant-\max _{0 \leqslant t \leqslant 1}|\phi(t)| \geqslant r \\
\kappa_{p}(\phi) \leqslant-\left(\frac{p^{p} r^{2}}{2 \Gamma(p+1) \sigma_{f}}\right)^{\frac{1}{2-p}},
\end{gathered}
$$

where

$$
\zeta_{n}(t)=\pi(g) \pi(\alpha)^{-1} \sum_{k=1}^{[n t]} I_{\alpha}\left(X_{k}\right), \quad 0 \leqslant t \leqslant 1, n=1,2, \ldots
$$

Consequently,

$$
\limsup _{n \rightarrow \infty} \frac{1}{b_{n}} \log P\left\{\max _{0 \leqslant t \leqslant 1}\left|\eta_{n}(t)-\zeta_{n}(t)\right| \geqslant \varepsilon a\left(\frac{n}{b_{n}}\right) b_{n}\right\}=-\infty
$$

for every $\varepsilon>0$. By Theorem 4.2.13 in Dembo and Zeitouni [15], our conclusion is true for any special function $g$.

By now, we have proved Theorem 1.1 in the atomic case.

Remark 3.1. To establish the Strassen invariance principle given in next section, we need to sharpen the lower bound into

$$
\liminf _{n \rightarrow \infty} \frac{1}{b_{n}} \log P_{\mu}\left\{\Xi_{n} \in \mathcal{O}, \tau_{\alpha} \circ \theta^{n} \leqslant \varepsilon n\right\} \geqslant-\inf _{(\phi, \gamma) \in \mathcal{O}} I_{p}(\phi, \gamma)
$$

for any open set $\mathcal{O} \subset D\left\{[0,1], \mathbf{R}^{2}\right\}$ and any $\varepsilon>0$. Indeed, from (3.8) we have

$$
\begin{array}{r}
\liminf _{n \rightarrow \infty} \frac{1}{b_{n}} \log E_{\mu}\left[\operatorname { e x p } \left\{\frac{1}{\sqrt{a\left(n b_{n}^{-1}\right)}} \sum_{k=1}^{m} A_{k}\left(\xi_{n_{k}}(1)-\xi_{n_{k-1}}(1)\right)\right.\right. \\
\left.\left.\quad+\frac{1}{a\left(n b_{n}^{-1}\right)} \sum_{k=1}^{m} B_{k}\left(i_{\alpha}\left(n_{k}\right)-i_{\alpha}\left(n_{k-1}\right)\right)\right\} \mid \tau_{\alpha} \circ \theta^{n} \leqslant \varepsilon n\right]
\end{array}
$$




$$
\geqslant \sum_{k=1}^{m}\left(t_{k}-t_{k-1}\right) \Lambda_{p}\left(A_{k}, B_{k}\right)
$$

On the other hand, taking $f=g=0$ in (3.8) gives

$$
\lim _{n \rightarrow \infty} \frac{1}{b_{n}} \log P_{\mu}\left\{\tau_{\alpha} \circ \theta^{n} \leqslant \varepsilon n\right\}=0 .
$$

Hence from (3.6), (3.10) and (3.11) we have, respectively,

$$
\begin{aligned}
& \limsup _{n \rightarrow \infty} \frac{1}{b_{n}} \log E_{\mu}\left[\operatorname { e x p } \left\{\frac{1}{\sqrt{a\left(n b_{n}^{-1}\right)}} \sum_{k=1}^{m} A_{k}\left(\xi_{n_{k}}(1)-\xi_{n_{k-1}}(1)\right)\right.\right. \\
& \left.\left.\quad+\frac{1}{a\left(n b_{n}^{-1}\right)} \sum_{k=1}^{m} B_{k}\left(i_{\alpha}\left(n_{k}\right)-i_{\alpha}\left(n_{k-1}\right)\right)\right\} \mid \tau_{\alpha} \circ \theta^{n} \leqslant \varepsilon n\right] \\
& \quad \leqslant \sum_{k=1}^{m}\left(t_{k}-t_{k-1}\right) \Lambda_{p}\left(A_{k}, B_{k}\right), \\
& \lim _{\varepsilon \rightarrow 0} \sup _{s \in[0,1]} \limsup _{n \rightarrow \infty} \frac{1}{b_{n}} \log P_{\mu}\left\{\sup _{s \leqslant t \leqslant s+\varepsilon}\left|\xi_{n}(t)-\xi_{n}(s)\right|>\delta \sqrt{a\left(n b_{n}^{-1}\right)} b_{n} \mid \tau_{\alpha} \circ \theta^{n} \leqslant \varepsilon n\right\}=-\infty
\end{aligned}
$$

and

$$
\lim _{\varepsilon \rightarrow 0} \sup _{s \in[0,1]} \limsup _{n \rightarrow \infty} \frac{1}{b_{n}} \log P_{\mu}\left\{\sup _{s \leqslant t \leqslant s+\varepsilon}\left|\eta_{n}(t)-\eta_{n}(s)\right|>\delta a\left(n b_{n}^{-1}\right) b_{n} \mid \tau_{\alpha} \circ \theta^{n} \leqslant \varepsilon n\right\}=-\infty .
$$

By carrying out the same procedure, we obtain the same moderate deviation for the conditional distributions:

$$
\mathcal{L}_{\mu}\left(\Xi_{n} \mid \tau_{\alpha} \circ \theta^{n} \leqslant \varepsilon n\right), \quad n=1,2, \ldots
$$

Its lower bound, together with (3.16), implies our claim in Remark 3.1.

Step 2. The small set case.

We now prove our result under the assumption that there exists $C \in \mathcal{E}^{+}$and a probability measure $v$ on $(E, \mathcal{E})$ such that

$$
P(x, A) \geqslant b I_{C}(x) v(A), \quad x \in E, A \in \mathcal{E} .
$$

According to the construction of split chain, which was initiated by Nummelin [28,29] and Athreya and Ney [4] (see also Meyn and Tweedie [26] and Duflo [18]), under the minorization (3.17) the Markov chain $\left\{X_{n}\right\}_{n} \geqslant 0$ can be augmented into (without changing distribution) a Harris recurrent Markov chain $\left\{\left(X_{n}, Y_{n}\right)\right\}_{n} \geqslant 0$ with state space $E \times\{0,1\}$ and the atom $\alpha^{*}=C \times\{1\}$. Further, $\left\{\left(X_{n}, Y_{n}\right)\right\}_{n \geqslant 0}$ has the same regularity. When viewed as the functions on $E \times\{0,1\}, f$ and $g$ are charge and special function, respectively, with respect to $\left\{\left(X_{n}, Y_{n}\right)\right\}_{n} \geqslant 0$. Therefore, what we established in the previous step remains valid under (3.17).

Step 3. The general case.

We finally come to the general case. According to Theorem 5.2.1 in Meyn and Tweedie [26], the following weaker version of (3.17) holds:

$$
P^{m}(x, A) \geqslant b I_{C}(x) v(A), \quad x \in E, A \in \mathcal{E}
$$

holds for some $m \geqslant 1$ with the possibility that $m$ can not be reduced to 1 . 
Our approach is resolvent approximation, which has been used in Chen [8,9]. Let $0<\lambda<1$ be fixed and let $\left\{\delta_{n}\right\}_{n \geqslant 1}$ be a sequence of i.i.d. Bernoulli random variables with common law

$$
P\left\{\delta_{1}=0\right\}=1-P\left\{\delta_{1}=1\right\}=\lambda .
$$

We assume independence between $\left\{\delta_{n}\right\}_{n \geqslant 1}$ and $\left\{X_{n}\right\}_{n \geqslant 0}$. Define the renew sequence $\left\{\sigma_{k}\right\}_{k \geqslant 0}$ :

$$
\sigma_{0}=0 \quad \text { and } \quad \sigma_{k+1}=\inf \left\{n>\sigma_{k} ; \delta_{n}=1\right\} .
$$

Then $\left\{\sigma_{k}-\sigma_{k-1}\right\}_{k \geqslant 1}$ is an i.i.d. sequence with the common law

$$
P\left\{\sigma_{1}=n\right\}=(1-\lambda) \lambda^{n-1}, \quad n=1,2, \ldots
$$

By (5.9) in de Acosta [1], the random sequence $\left\{X_{\sigma_{n}}\right\}_{n \geqslant 0}$ is a Markov chain with the transition

$$
P_{\lambda}(x, A)=(1-\lambda) \sum_{k=1}^{\infty} \lambda^{k-1} P^{k}(x, A), \quad x \in E, A \in \mathcal{E}
$$

which is Harris recurrent with the same regularity. As a matter of fact, any $D$-set $D$ of $P$ is also a $D$-set of $P_{\lambda}$ with

$$
a_{\lambda}(n) \equiv \sum_{k=1}^{n} \nu P_{\lambda}^{k}(D) \sim(1-\lambda)^{1-p} a(n) \quad(n \rightarrow \infty),
$$

which can be easily shown by taking the expectations and passing to the limit in the following equality

$$
\sum_{k=1}^{n} 1_{D}\left(X_{\sigma(k)}\right)=\sum_{k=1}^{\sigma(n)} \delta_{k} 1_{D}\left(X_{k}\right)
$$

for some $D$-set $D$. Clearly, $P_{\lambda}$ has the same invariant measure $\pi$. Further, $f$ and $g$ are charge and special function, respectively, with respect to $P_{\lambda}$ and,

$$
\begin{aligned}
\sigma_{f, \lambda}^{2} & \equiv \int f^{2}(x) \pi(d x)+2 \sum_{k=1}^{\infty} \int f(x) P_{\lambda}^{k} f(x) \pi(d x) \\
& =\int f^{2}(x) \pi(d x)+2(1-\lambda) \sum_{k=1}^{\infty} \int f(x) P^{k} f(x) \pi(d x) .
\end{aligned}
$$

In view of (3.18), the transition $P_{\lambda}$ satisfies (3.17). Define

$$
\begin{aligned}
& \bar{\xi}_{n}(t)=\sum_{k=1}^{[n t]} f\left(X_{\sigma_{k}}\right), \quad 0 \leqslant t \leqslant 1, n=1,2, \ldots, \\
& \bar{\eta}_{n}(t)=\sum_{k=1}^{[n t]} g\left(X_{\sigma_{k}}\right), \quad 0 \leqslant t \leqslant 1, n=1,2, \ldots,
\end{aligned}
$$

and

$$
\bar{\Xi}_{n}(t)=\left(\frac{1}{\sqrt{a\left(n b_{n}^{-1}\right) b_{n}}} \bar{\xi}_{n}(t), \frac{1}{a\left(n b_{n}^{-1}\right) b_{n}} \bar{\eta}_{n}(t)\right), \quad 0 \leqslant t \leqslant 1 .
$$

Applying what has been proved in Step 2 to the resolvent chain $P_{\lambda}$ and taking (3.19) and (3.20) into account give that for each closed set $\mathcal{C} \in D\left\{[0,1], \mathbf{R}^{2}\right\}$

$$
\limsup _{n \rightarrow \infty} \frac{1}{b_{n}} \log P\left\{\bar{\Xi}_{n}(\cdot) \in \mathcal{C}\right\} \leqslant-\inf _{(\phi, \gamma) \in \mathcal{C}} \bar{I}_{p}(\phi, \gamma)
$$


and, for each open set $\mathcal{O} \in D\left\{[0,1], \mathbf{R}^{2}\right\}$

$$
\liminf _{n \rightarrow \infty} \frac{1}{b_{n}} \log P\left\{\bar{\Xi}_{n}(\cdot) \in \mathcal{O}\right\} \geqslant-\inf _{(\phi, \gamma) \in \mathcal{O}} \bar{I}_{p}(\phi, \gamma)
$$

where

$$
\bar{I}_{p}(\phi, \gamma)= \begin{cases}\int_{0}^{1} \bar{\Lambda}_{p}^{*}(\dot{\phi}(t), \dot{\gamma}(t)) d t & \text { if }(\phi, \gamma) \in \Theta_{p}, \\ +\infty & \text { otherwise }\end{cases}
$$

with $\bar{\Lambda}_{p}^{*}$ being given as

$$
\bar{\Lambda}_{p}^{*}(x, y)=\frac{\pi(g) x^{2}}{2 \sigma_{f, \lambda}^{2} y}+(1-p)\left(\frac{p^{p} y}{\pi(g)(1-\lambda)^{p} \Gamma(p+1)}\right)^{(1-p)^{-1}}, \quad(x, y) \in \mathbf{R} \times \mathbf{R}^{+} .
$$

Let

$$
\begin{aligned}
& \hat{\xi}_{n}(t)=\sum_{k=1}^{[n t]} \delta_{k} f\left(X_{k}\right), \quad 0 \leqslant t \leqslant 1, n=1,2, \ldots, \\
& \hat{\eta}_{n}(t)=\sum_{k=1}^{[n t]} \delta_{k} g\left(X_{k}\right), \quad 0 \leqslant t \leqslant 1, n=1,2, \ldots
\end{aligned}
$$

We claim that for any $\delta>0$,

$$
\begin{aligned}
& \limsup _{n \rightarrow \infty} \frac{1}{b_{n}} \log P\left\{\max _{0 \leqslant t \leqslant 1}\left|\bar{\xi}_{[n(1-\lambda)]}(t)-\hat{\xi}_{n}(t)\right| \geqslant \delta \sqrt{a\left(n b_{n}^{-1}\right)} b_{n}\right\}=-\infty, \\
& \limsup _{n \rightarrow \infty} \frac{1}{b_{n}} \log P\left\{\max _{0 \leqslant t \leqslant 1}\left|\bar{\eta}_{[n(1-\lambda)]}(t)-\hat{\eta}_{n}(t)\right| \geqslant \delta a\left(n b_{n}^{-1}\right) b_{n}\right\}=-\infty .
\end{aligned}
$$

Due to the similarity, we only prove (3.23). Write

$$
l(0)=0 \quad \text { and } \quad l(n)=\delta_{1}+\cdots+\delta_{n}, \quad n=1,2, \ldots
$$

Notice that,

$$
\hat{\xi}_{n}(t)=\sum_{i=1}^{l([n t])} f\left(X_{\sigma_{i}}\right), \quad 0 \leqslant t \leqslant 1, n=1,2, \ldots
$$

We have that for any $0<\varepsilon<\lambda$,

$$
\begin{aligned}
\max _{0 \leqslant t \leqslant 1}\left|\bar{\xi}_{[n(1-\lambda)]}(t)-\hat{\xi}_{n}(t)\right| & \leqslant \max _{0 \leqslant t \leqslant 1}\left|\sum_{i=1}^{[n(1-\lambda) t]} f\left(X_{\sigma_{i}}\right)-\sum_{i=1}^{l([n t])} f\left(X_{\sigma_{i}}\right)\right| \\
& \leqslant \max _{1 \leqslant j \leqslant[n(1-\lambda)]} \max _{j \leqslant k \leqslant j+\varepsilon n}\left|\sum_{i=j+1}^{k} f\left(X_{\sigma_{i}}\right)\right| \\
& \leqslant \max _{|s-t| \leqslant \varepsilon}\left|\bar{\xi}_{n}(s)-\bar{\xi}_{n}(t)\right|
\end{aligned}
$$

on the event $\left\{\max _{0 \leqslant t \leqslant 1}|l([n t])-[n(1-\lambda) t]| \leqslant \varepsilon n\right\}$. 
On the other hand, by Mogulskii's functional large deviation (see, cf., Theorem 5.1.2 in Dembo and Zeitouni [15]), there exist $u=u(\varepsilon)>0$, such that

$$
P\left\{\max _{0 \leqslant t \leqslant 1}|l([n t])-[n(1-\lambda) t]| \geqslant \varepsilon n\right\} \leqslant \mathrm{e}^{-u n}
$$

for sufficiently large $n$.

To have (3.23), therefore, we only need

$$
\lim _{\varepsilon \rightarrow 0} \limsup _{n \rightarrow \infty} \frac{1}{b_{n}} \log P\left\{\max _{|s-t| \leqslant \varepsilon}\left|\bar{\xi}_{n}(s)-\bar{\xi}_{n}(t)\right| \geqslant \delta \sqrt{a\left(n b_{n}^{-1}\right)} b_{n}\right\}=-\infty .
$$

Indeed, taking $\mathcal{C}=\left\{(\phi, \gamma): \max _{|s-t| \leqslant \varepsilon}\left|\bar{\xi}_{n}(s)-\bar{\xi}_{n}(t)\right| \geqslant \delta\right\}$ in (3.21) gives, in view of (1.15), that

$$
\begin{aligned}
& \limsup _{n \rightarrow \infty} \frac{1}{b_{n}} \log P\left\{\max _{|s-t| \leqslant \varepsilon}\left|\bar{\xi}_{n}(s)-\bar{\xi}_{n}(t)\right| \geqslant \delta \sqrt{a\left(n b_{n}^{-1}\right)} b_{n}\right\} \\
& \leqslant-\max _{|s-t| \leqslant \varepsilon} \inf _{\phi(s)-\phi(t) \mid \geqslant \delta} \int_{0}^{1}\left(\frac{p^{p} \dot{\phi}^{2}(t)}{2(1-\lambda)^{p} \Gamma(p+1) \sigma_{f, \lambda}^{2}}\right)^{\frac{1}{2-p}} d t \\
& \quad \leqslant-\left(\frac{p^{p} \delta^{2} \varepsilon^{-p}}{2(1-\lambda)^{p} \Gamma(p+1) \sigma_{f, \lambda}^{2}}\right)^{\frac{1}{2-p}}
\end{aligned}
$$

which leads to (3.25).

Let

$$
\widehat{\Xi}_{n}(\cdot)=\left(\frac{1}{\sqrt{a\left(n b_{n}^{-1}\right) b_{n}}} \hat{\xi}_{n}(\cdot), \frac{1}{a\left(n b_{n}^{-1}\right) b_{n}} \hat{\eta}_{n}(\cdot)\right), \quad n=1,2, \ldots
$$

According to Theorem 4.2.13 in Dembo and Zeitouni [15], (3.22) and (3.23) imply that $\left\{\widehat{\Xi}_{n}\right\}$ obeys the same moderate deviation that holds for $\left\{\bar{\Xi}_{[(1-\lambda) n]}\right\}$. By (3.21) and (3.22) (with $n$ being replaced by $[n(1-\lambda)]$ ), therefore, for each closed set $\mathcal{C} \in D\left\{[0,1], \mathbf{R}^{2}\right\}$

$$
\limsup _{n \rightarrow \infty} \frac{1}{b_{n}} \log P\left\{\widehat{\Xi}_{n}(\cdot) \in \mathcal{C}\right\} \leqslant-\inf _{(\phi, \gamma) \in \mathcal{C}} \hat{I}_{p}(\phi, \gamma)
$$

and, for each open set $\mathcal{O} \in D\left\{[0,1], \mathbf{R}^{2}\right\}$

$$
\liminf _{n \rightarrow \infty} \frac{1}{b_{n}} \log P\left\{\widehat{\Xi}_{n}(\cdot) \in \mathcal{O}\right\} \geqslant-\inf _{(\phi, \gamma) \in \mathcal{O}} \hat{I}_{p}(\phi, \gamma)
$$

where

$$
\hat{I}_{p}(\phi, \gamma)= \begin{cases}\int_{0}^{1} \hat{\Lambda}_{p}^{*}(\dot{\phi}(t), \dot{\gamma}(t)) d t & \text { if }(\phi, \gamma) \in \Theta_{p}, \\ +\infty & \text { otherwise }\end{cases}
$$

with $\hat{\Lambda}_{p}^{*}$ being given as

$$
\hat{\Lambda}_{p}^{*}(x, y)=\frac{\pi(g) x^{2}}{2 \sigma_{f, \lambda}^{2} y}+(1-p)\left(\frac{p^{p} y}{\pi(g)(1-\lambda) \Gamma(p+1)}\right)^{(1-p)^{-1}} \quad(x, y) \in \mathbf{R} \times \mathbf{R}^{+} .
$$


Let

$$
\begin{aligned}
& \tilde{\xi}_{n}(t)=\sum_{k=1}^{[n t]}\left(1-\delta_{k}\right) f\left(X_{k}\right), \quad 0 \leqslant t \leqslant 1, n=1,2, \ldots, \\
& \tilde{\eta}_{n}(t)=\sum_{k=1}^{[n t]}\left(1-\delta_{k}\right) g\left(X_{k}\right), \quad 0 \leqslant t \leqslant 1, n=1,2, \ldots .
\end{aligned}
$$

Replacing $\left\{\delta_{n}\right\}_{n} \geqslant 1$ by $\left\{1-\delta_{n}\right\}_{n} \geqslant 1$ gives us the same moderate deviation as described in (2.25) and (2.26), except that $\lambda$ is replaced by $1-\lambda$ in the construction of the rate function. Applying its upper bound to the set

$$
\left\{(\phi, \gamma): \max _{0 \leqslant t \leqslant 1}|\phi(t)| \geqslant \varepsilon\right\}
$$

and taking (1.15) into account we have that for any $\varepsilon>0$,

$$
\begin{aligned}
& \limsup _{n \rightarrow \infty} \frac{1}{b_{n}} \log P\left\{\max _{0 \leqslant t \leqslant 1}\left|\tilde{\xi}_{n}(t)\right| \geqslant \varepsilon \sqrt{a\left(n b_{n}^{-1}\right)} b_{n}\right\} \\
& =-(2-p) \max _{0 \leqslant t \leqslant 1}|\phi(t)| \geqslant \varepsilon \\
& \inf _{0}\left(\frac{p^{p} \dot{\phi}^{2}(t)}{2 \lambda \Gamma(p+1) \sigma_{f, 1-\lambda}^{2}}\right)^{\frac{1}{2-p}} d t \\
& \leqslant-\left(\frac{p^{p} \varepsilon^{2}}{2 \lambda \Gamma(p+1) \sigma_{f, 1-\lambda}^{2}}\right)^{\frac{1}{2-p}} .
\end{aligned}
$$

Consequently,

$$
\limsup _{\lambda \rightarrow 0^{+}} \limsup _{n \rightarrow \infty} \frac{1}{b_{n}} \log P\left\{\max _{0 \leqslant t \leqslant 1}\left|\tilde{\xi}_{n}(t)\right| \geqslant \varepsilon \sqrt{a\left(n b_{n}^{-1}\right)} b_{n}\right\}=-\infty .
$$

Similarly,

$$
\limsup _{\lambda \rightarrow 0^{+}} \limsup _{n \rightarrow \infty} \frac{1}{b_{n}} \log P\left\{\max _{0 \leqslant t \leqslant 1}\left|\tilde{\eta}_{n}(t)\right| \geqslant \varepsilon a\left(n b_{n}^{-1}\right) b_{n}\right\}=-\infty .
$$

Notice that

$$
\xi_{n}(\cdot)=\hat{\xi}_{n}(\cdot)+\tilde{\xi}_{n}(\cdot) \quad \text { and } \quad \eta_{n}(\cdot)=\hat{\eta}_{n}(\cdot)+\tilde{\eta}_{n}(\cdot), \quad n=1,2, \ldots
$$

By Theorem 4.2.16 in Dembo and Zeitouni [15], Theorem 1.1 follows from (3.27)-(3.31), the convergence of the rate functions being here readily done as $\sigma_{f, \lambda}^{2} \rightarrow \sigma_{f}^{2}$ when $\lambda \rightarrow 0$.

\section{A functional law of the iterated logarithm}

We now apply our results to obtain a functional law of the iterated logarithm. From now on, we take $b_{n}=\log \log n$. Recall that

$$
\Xi_{n}(\cdot)=\left(\xi_{n}(\cdot) / \sqrt{a\left(\frac{n}{\log \log n}\right)} \log \log n, \eta_{n}(\cdot) / a\left(\frac{n}{\log \log n}\right) \log \log n\right), \quad n=1,2, \ldots
$$

We also need some related notations. Given a metric space $\mathcal{X}$ with the distance $d(\cdot, \cdot)$ and sequence $x_{n} \in \mathcal{X}$ $(n \geqslant 1)$, we use $C\left(\left\{x_{n}\right\}\right)$ to denote the cluster set of the sequence $\left\{x_{n}\right\}$. Given a subset $K \subset \mathcal{X}$, we say that $\left\{x_{n}\right\}$ converges to $K$, or write

$$
x_{n} \rightarrow K
$$


if

$$
C\left(\left\{x_{n}\right\}\right)=K \quad \text { and } \quad \lim _{n \rightarrow \infty} d\left(x_{n}, K\right)=0
$$

where $d(x, K)=\inf _{y \in K} d(x, y)$.

Given a rate function $I$ on $B$, we write

$$
K(I)=\{x \in B ; I(x) \leqslant 1\} .
$$

Theorem 4.1. Assume that the Harris recurrent Markov chain $\left\{X_{n}\right\}_{n \geqslant 0}$ is p-regular and that $f$ is a charge, $g$ is special.

(1) When $0<p<1$,

$$
\begin{aligned}
& \Xi_{n}(\cdot) \rightarrow K\left(I_{p}\right) \quad \text { a.s. } \\
& \eta_{n}(\cdot) / a\left(\frac{n}{\log \log n}\right) \log \log n \stackrel{\text { a.s. }}{\longrightarrow} K\left(J_{p}\right) .
\end{aligned}
$$

(2) When $0<p \leqslant 1$,

$$
\xi_{n}(\cdot) / \sqrt{a\left(\frac{n}{\log \log n}\right)} \log \log n \stackrel{\text { a.s. }}{\longrightarrow} K\left(\kappa_{p}\right) .
$$

Remark 4.2. According to Theorem 17.3.2 in Meyn and Tweedie [26], the algebra $\mathcal{A}$ of the sets invariant under the shifting $\theta$ is almost surely trivial when $\left\{X_{n}\right\}_{n \geqslant 0}$ is Harris recurrent: For every $A \in \mathcal{A}, P_{\mu}(A)=1$ for every initial distribution $\mu$, or $P_{\mu}(A)=0$ for every initial distribution $\mu$. Consequently, the strong laws stated in Theorem 4.1 and in the theorems given in the next section are independent of the choice of initial distribution $\mu$.

Proof of Theorem 4.1. Due to similarity, we only prove (4.1). Fix $\rho>1$ and let $n_{k}=\left[\rho^{k}\right]$. Write

$$
c_{k}=\sqrt{a\left(\frac{n_{k}}{\log \log n_{k}}\right)} \log \log n_{k} \quad \text { and } \quad d_{k}=a\left(\frac{n_{k}}{\log \log n_{k}}\right) \log \log n_{k}
$$

where $k=1,2, \ldots$

We use $\|\cdot\|$ to denote the sup-norm in $D[0,1]$, i.e.,

$$
\|\phi\|=\sup _{0 \leqslant t \leqslant 1}|\phi(t)|, \quad \phi \in D[0,1] .
$$

Without causing any confusion, we also use $\|\cdot\|$ for the norm on $D\left\{[0,1], \mathbf{R}^{2}\right\}$, i.e.,

$$
\|(\phi, \gamma)\|=\|\phi\|+\|\gamma\|, \quad(\phi, \gamma) \in D\left\{[0,1], \mathbf{R}^{2}\right\} .
$$

We first show that

$$
\lim _{n \rightarrow \infty} d\left(\Xi_{n}, K\left(I_{p}\right)\right)=0 \text { a.s. }
$$

By the upper bounds given in Theorem 1.1, for any $\varepsilon>0$,

$$
\limsup _{n \rightarrow \infty} \frac{1}{\log \log n} \log P_{\mu}\left\{d\left(\Xi_{n}, K\left(I_{p}\right)\right) \geqslant \varepsilon\right\} \leqslant-\inf _{d\left((\phi, \gamma), K\left(I_{p}\right)\right) \geqslant \varepsilon} I_{p}(\phi, \gamma)<-1 .
$$

Consequently,

$$
\sum_{k} P_{\mu}\left\{d\left(\Xi_{n_{k}}, K\left(I_{p}\right)\right) \geqslant \varepsilon\right\}<\infty .
$$


By Borel-Cantelli lemma,

$$
\lim _{k \rightarrow \infty} d\left(\Xi_{n_{k}}, K\left(I_{p}\right)\right)=0 \quad \text { a.s. }
$$

Given $n_{k} \leqslant n \leqslant n_{k+1}$,

$$
\left\|\Xi_{n}-\Xi_{n_{k}}\right\| \leqslant \frac{1}{c_{k}}\left\|\xi_{n}-\xi_{n_{k}}\right\|+\frac{1}{d_{k}}\left\|\eta_{n}-\eta_{n_{k}}\right\|+\left(\frac{c_{k+1}}{c_{k}}-1\right) \frac{1}{c_{k+1}}\left\|\xi_{n_{k+1}}\right\|+\left(\frac{d_{k+1}}{d_{k}}-1\right) \frac{1}{d_{k+1}}\left\|\eta_{n_{k+1}}\right\| .
$$

By (4.5), there is a constant $C>0$ independent of $\rho>1$ such that

$$
\limsup _{k \rightarrow \infty} \frac{1}{c_{k+1}}\left\|\xi_{n_{k+1}}\right\| \leqslant C \quad \text { and } \quad \limsup _{k \rightarrow \infty} \frac{1}{d_{k+1}}\left\|\eta_{n_{k+1}}\right\| \leqslant C \quad \text { a.s. }
$$

Notice that

$$
\left\|\xi_{n}-\xi_{n_{k}}\right\| \leqslant \max _{|s-t| \leqslant \varepsilon}\left|\xi_{n_{k+1}}(s)-\xi_{n_{k+1}}(t)\right|
$$

eventually holds for any $\varepsilon>1-\rho^{-1}$ and the simple fact that

$$
\inf \left\{\kappa_{p}(\phi) ; \max _{|s-t| \leqslant \varepsilon}|\phi(s)-\phi(t)| \geqslant \delta\right\} \rightarrow \infty
$$

as $\varepsilon \rightarrow 0$. By the upper bound in Theorem 1.3, therefore, given $\delta>0$, we have

$$
\limsup _{k \rightarrow \infty} \frac{1}{\log \log n_{k}} \log P_{\mu}\left\{\max _{n_{k} \leqslant n \leqslant n_{k+1}}\left\|\xi_{n}-\xi_{n_{k}}\right\| \geqslant \delta c_{k}\right\}<-1
$$

as $\rho>1$ is sufficiently close to 1 , see upper bound (3.26). Therefore,

$$
\sum_{k} P_{\mu}\left\{\max _{n_{k} \leqslant n \leqslant n_{k+1}}\left\|\xi_{n}-\xi_{n_{k}}\right\| \geqslant \delta c_{k}\right\}<\infty
$$

By Borel-Cantelli lemma,

$$
\limsup _{k \rightarrow \infty} \frac{1}{c_{k}} \max _{n_{k} \leqslant n \leqslant n_{k+1}}\left\|\xi_{n}-\xi_{n_{k}}\right\| \leqslant \delta \quad \text { a.s. }
$$

Similarly, as $\rho>1$ is sufficiently close to 1 we have

$$
\limsup _{k \rightarrow \infty} \frac{1}{d_{k}} \max _{n_{k} \leqslant n \leqslant n_{k+1}}\left\|\eta_{n}-\eta_{n_{k}}\right\| \leqslant \delta \quad \text { a.s. }
$$

Summarizing what we have observed, we obtain

$$
\max _{n_{k} \leqslant n \leqslant n_{k+1}}\left\|\Xi_{n}-\Xi_{n_{k}}\right\| \leqslant 2 \delta+2(\rho-1) C \quad \text { a.s. }
$$

In view of (4.5), we have

$$
\limsup _{n \rightarrow \infty} d\left(\Xi_{n}, K\left(I_{p}\right)\right) \leqslant 2 \delta+2(\rho-1) C \text { a.s. }
$$

Letting $\rho \rightarrow 1^{+}$and then $\delta \rightarrow 0^{+}$gives (4.4).

From (4.4) we have that $C\left(\left\{\Xi_{n}\right\}\right) \subset K\left(I_{p}\right)$ a.s. Notice that $\left\{(\phi, \gamma) ; I_{p}(\phi, \gamma)<1\right\}$ is dense in $K\left(I_{p}\right)$. It remains to prove that for any $(\phi, \gamma) \in D\left\{[0,1], \mathbf{R}^{2}\right\}$ with $I_{p}(\phi, \gamma)<1$,

$$
\liminf _{n \rightarrow \infty}\left\|\Xi_{n}-(\phi, \gamma)\right\|=0 \quad \text { a.s. }
$$

In view of the procedure we carry out in Section 3, which extends the moderate deviation from the atomic case to its full generality, we may assume that $\left\{X_{n}\right\}_{n} \geqslant 0$ has an atom $\alpha$. Let $\delta>0$ be fixed and define

$$
A_{k}=\left\{\left\|\frac{1}{c_{k}} \xi_{n_{k}}-\phi\right\|<\delta,\left\|\frac{1}{d_{k}} \eta_{n_{k}}-\gamma\right\|<\delta, \tau_{\alpha} \circ \theta^{n_{k}} \leqslant n_{k}\right\}, \quad k=1,2, \ldots
$$


By Remark 3.1,

$$
\liminf _{k \rightarrow \infty} \frac{1}{\log \log n_{k}} \log P_{\alpha}\left(A_{k}\right) \geqslant-I_{p}(\phi, \gamma)>-1 .
$$

Hence

$$
\sum_{k} P_{\alpha}\left(A_{k}\right)=\infty
$$

Given $\lambda>0$, define

$$
T=\inf \left\{k ; \sum_{j=1}^{k} I_{A_{j}} \geqslant \lambda\right\}
$$

Then for any integer $N \geqslant 1$, real number $M>0$,

$$
\begin{aligned}
P_{\alpha}\left\{\sum_{k=1}^{N} I_{A_{k}} \geqslant 3 \lambda+M+1\right\} & =P_{\alpha}\left\{\sum_{k=1}^{N} I_{A_{k}} \geqslant 3 \lambda+M+1, T \leqslant N\right\} \\
& =\sum_{j=1}^{N} P_{\alpha}\left\{\sum_{k=1}^{N} I_{A_{k}} \geqslant 3 \lambda+M+1, T=j\right\} \\
& \leqslant \sum_{j=1}^{N-1} P_{\alpha}\left\{T=j, \sum_{k=j+1}^{N} I_{A_{k}} \geqslant 2 \lambda+M\right\} .
\end{aligned}
$$

For any $j<k \leqslant N$, notice that

$$
\xi_{n_{k}}(t) \circ \theta^{n_{j}+\tau_{\alpha} \circ \theta^{n_{j}}}=\sum_{i=1+n_{j}+\tau_{\alpha} \circ \theta^{n_{j}}}^{n_{j}+\tau_{\alpha} \circ \theta^{n_{j}}+\left[n_{k} t\right]} f\left(X_{i}\right), \quad 0 \leqslant t \leqslant 1 .
$$

Hence, on the event $\left\{\tau_{\alpha} \circ \theta^{n_{j}} \leqslant n_{j}\right\}$,

$$
\begin{aligned}
\left\|\xi_{n_{k}} \circ \theta^{n_{j}+\tau_{\alpha} \circ \theta^{n_{j}}}-\xi_{n_{k}}\right\| & \leqslant\left|\sum_{i=1}^{n_{j}+\tau_{\alpha} \circ \theta^{n_{j}}} f\left(X_{i}\right)\right|+\max _{0 \leqslant t \leqslant 1}\left|\sum_{i=\left[n_{k} t\right]+1}^{n_{j}+\tau_{\alpha} \circ \theta^{n_{j}}+\left[n_{k} t\right]} f\left(X_{i}\right)\right| \\
& \leqslant 2 \max _{|t-s| \leqslant 2 \rho^{-1}}\left|\xi_{2 n_{k}}(t)-\xi_{2 n_{k}}(s)\right|
\end{aligned}
$$

as $\rho>2$, where the second step follows from the estimate

$$
\left(n_{j}+\tau_{\alpha} \circ \theta^{n_{j}}+\left[n_{k} t\right]\right)-\left[n_{k} t\right]=n_{j}+\tau_{\alpha} \circ \theta^{n_{j}} \leqslant 2 n_{j} \leqslant 2 \rho^{-1} n_{k}
$$

for all $k \geqslant j+1$.

Similarly,

$$
\left\|\eta_{n_{k}} \circ \theta^{n_{j}+\tau_{\alpha} \circ \theta^{n_{j}}}-\eta_{n_{k}}\right\| \leqslant 2 \max _{|t-s| \leqslant 2 \rho^{-1}}\left|\eta_{2 n_{k}}(t)-\eta_{2 n_{k}}(s)\right| .
$$

Define

$$
\begin{aligned}
& B_{k}=\left\{\left\|\frac{1}{c_{k}} \xi_{n_{k}}-\phi\right\|<4 \delta,\left\|\frac{1}{d_{k}} \eta_{n_{k}}-\gamma\right\|<4 \delta\right\}, \\
& \widetilde{B}_{k}=\left\{\left\|\frac{1}{c_{k}} \xi_{n_{k}} \circ \theta^{n_{j}+\tau_{\alpha} \circ \theta^{n_{j}}}-\phi\right\|<4 \delta,\left\|\frac{1}{d_{k}} \eta_{n_{k}} \circ \theta^{n_{j}+\tau_{\alpha} \circ \theta^{n_{j}}}-\gamma\right\|<4 \delta\right\},
\end{aligned}
$$




$$
\begin{aligned}
& C_{k}=\left\{\max _{|t-s| \leqslant 2 \rho^{-1}}\left|\xi_{2 n_{k}}(t)-\xi_{2 n_{k}}(s)\right| \geqslant \delta c_{k}\right\}, \\
& D_{k}=\left\{\max _{|t-s| \leqslant 2 \rho^{-1}}\left|\eta_{2 n_{k}}(t)-\eta_{2 n_{k}}(s)\right| \geqslant \delta d_{k}\right\},
\end{aligned}
$$

where $k=1,2, \ldots$. Notice that

$$
\{T=j\} \subset A_{j} \subset\left\{\tau_{\alpha} \circ \theta^{n_{j}} \leqslant n_{j}\right\} .
$$

From the above discussion, for any $j<k \leqslant N$,

$$
\begin{aligned}
& P_{\alpha}\left\{T=j, \sum_{k=j+1}^{N} I_{A_{k}} \geqslant 2 \lambda+M\right\} \\
& \quad \leqslant P_{\alpha}\left\{T=j, \sum_{k=j+1}^{N} I_{\widetilde{B}_{k} \cup C_{k} \cup D_{k}} \geqslant 2 \lambda+M\right\} \\
& \quad \leqslant P_{\alpha}\left\{T=j, \sum_{k=j+1}^{N} I_{\widetilde{B}_{k}} \geqslant M\right\}+P_{\alpha}\left\{T=j, \sum_{k=j+1}^{N} I_{C_{k}} \geqslant \lambda\right\}+P_{\alpha}\left\{T=j, \sum_{k=j+1}^{N} I_{D_{k}} \geqslant \lambda\right\} .
\end{aligned}
$$

Notice that by Markov property the first term on the right hand side is equal to

$$
P_{\alpha}\{T=j\} P_{\alpha}\left\{\sum_{k=j+1}^{N} I_{B_{k}} \geqslant M\right\} \leqslant P_{\alpha}\{T=j\} P_{\alpha}\left\{\sum_{k=1}^{\infty} I_{B_{k}} \geqslant M\right\} .
$$

Thus, for any $\lambda>0$

$$
\begin{aligned}
& P_{\alpha}\left\{\sum_{k=1}^{N} I_{A_{k}} \geqslant 3 \lambda+M+1\right\} \\
& \leqslant P_{\alpha}\{T \leqslant N\} P_{\alpha}\left\{\sum_{k=1}^{\infty} I_{B_{k}} \geqslant M\right\}+\sum_{j=1}^{N} P_{\alpha}\left\{T=j, \sum_{k=1}^{\infty} I_{C_{k}} \geqslant \lambda\right\}+\sum_{j=1}^{N} P_{\alpha}\left\{T=j, \sum_{k=1}^{\infty} I_{D_{k}} \geqslant \lambda\right\} \\
& \quad \leqslant P_{\alpha}\left\{\sum_{k=1}^{N} I_{A_{k}} \geqslant \lambda\right\} P_{\alpha}\left\{\sum_{k=1}^{\infty} I_{B_{k}} \geqslant M\right\}+P_{\alpha}\left\{\sum_{k=1}^{\infty} I_{C_{k}} \geqslant \lambda\right\}+P_{\alpha}\left\{\sum_{k=1}^{\infty} I_{D_{k}} \geqslant \lambda\right\} .
\end{aligned}
$$

Therefore

$$
\begin{aligned}
\sum_{k=1}^{N} P_{\alpha}\left(A_{k}\right)= & \int_{0}^{\infty} P_{\alpha}\left\{\sum_{k=1}^{N} I_{A_{k}} \geqslant \lambda\right\} d \lambda \\
\leqslant & M+1+3 \int_{0}^{\infty} P_{\alpha}\left\{\sum_{k=1}^{N} I_{A_{k}} \geqslant 3 \lambda+M+1\right\} d \lambda \\
\leqslant & M+1+3 P_{\alpha}\left\{\sum_{k=1}^{\infty} I_{B_{k}} \geqslant M\right\} \int_{0}^{\infty} P_{\alpha}\left\{\sum_{k=1}^{N} I_{A_{k}} \geqslant \lambda\right\} d \lambda \\
& +3 \int_{0}^{\infty} P_{\alpha}\left\{\sum_{k=1}^{\infty} I_{C_{k}} \geqslant \lambda\right\} d \lambda+3 \int_{0}^{\infty} P_{\alpha}\left\{\sum_{k=1}^{\infty} I_{D_{k}} \geqslant \lambda\right\} d \lambda
\end{aligned}
$$




$$
=M+1+3 P_{\alpha}\left\{\sum_{k=1}^{\infty} I_{B_{k}} \geqslant M\right\} \sum_{k=1}^{N} P_{\alpha}\left(A_{k}\right)+3 \sum_{k=1}^{\infty} P_{\alpha}\left(C_{k}\right)+3 \sum_{k=1}^{\infty} P_{\alpha}\left(D_{k}\right) .
$$

Hence we have

$$
\left(1-3 P_{\alpha}\left\{\sum_{k=1}^{\infty} I_{B_{k}} \geqslant M\right\}\right) \sum_{k=1}^{N} P_{\alpha}\left(A_{k}\right) \leqslant M+1+3 \sum_{k=1}^{\infty} P_{\alpha}\left(C_{k}\right)+3 \sum_{k=1}^{\infty} P_{\alpha}\left(D_{k}\right) .
$$

On the other hand, as $f \rightarrow \sup _{|t-s|<\delta}|f(t)-f(s)|$ is a continuous mapping, by the upper bounds in Theorems 1.2 and 1.3, one can see that as $\rho>1$ is sufficiently large,

$$
\limsup _{k \rightarrow \infty} \frac{1}{\log \log n_{k}} \log P_{\alpha}\left(C_{k}\right)<-1 \text { and } \limsup _{k \rightarrow \infty} \frac{1}{\log \log n_{k}} \log P_{\alpha}\left(D_{k}\right)<-1,
$$

indeed, use the upper bound (3.26). Consequently,

$$
\sum_{k=1}^{\infty} P_{\alpha}\left(C_{k}\right)<\infty \text { and } \sum_{k=1}^{\infty} P_{\alpha}\left(D_{k}\right)<\infty .
$$

In view of (4.7) we must have

$$
P_{\alpha}\left\{\sum_{k=1}^{\infty} I_{B_{k}} \geqslant M\right\} \geqslant \frac{1}{3}
$$

Let $M \rightarrow \infty$ gives that

$$
P_{\alpha}\left\{\left\|\frac{1}{c_{k}} \xi_{n_{k}}-\phi\right\|<4 \delta,\left\|\frac{1}{d_{k}} \eta_{n_{k}}-\gamma\right\|<4 \delta \text { i.o. }\right\} \geqslant \frac{1}{3} .
$$

Notice that $\delta>0$ can be arbitrarily small. We have that

$$
P_{\alpha}\left\{\liminf _{n \rightarrow \infty}\left\|\Xi_{n}-(\phi, \gamma)\right\|=0\right\} \geqslant \frac{1}{3} .
$$

By Theorem 17.3.2 in Meyn and Tweedie [25], (4.6) holds.

\section{The LIL for some interesting functionals}

Given a continuous map $\psi$ from a metric space $\mathcal{X}$ to another metric space $\mathcal{Y}$ and $x_{n} \in \mathcal{X}(n \geqslant 1)$ with $x_{n} \rightarrow K$ for some $K \subset \mathcal{X}$, we have $\psi\left(x_{n}\right) \rightarrow \psi(K)$. If $\psi$ is a continuous function on $B$, then

$$
\limsup _{n \rightarrow \infty} \psi\left(x_{n}\right)=\sup _{x \in K} \psi(x) \text {. }
$$

By considering various functionals, we obtain some direct consequences from Theorem 4.1. First, we notice that the two dimensional random variable $\Xi_{n}(1)$ takes values in the metric space $\mathcal{X}=(-\infty,+\infty) \times[0, \infty)$. By considering the continuous map $(\phi, \gamma) \mapsto(\phi(1), \gamma(1))$ we have

Theorem 5.1. Assume that the Harris recurrent Markov chain $\left\{X_{n}\right\}_{n} \geqslant 0$ is $p$-regular with $0<p<1$ and that $f$ is a charge, $g$ is special. Then

$$
\Xi_{n}(1) \rightarrow K\left(\Lambda_{p}^{*}\right) \quad \text { a.s. }
$$

Consequently, for each $\beta \leqslant 1 / 2$, 


$$
\begin{aligned}
& \limsup _{n \rightarrow \infty} a\left(\frac{n}{\log \log n}\right)^{-\frac{1}{2}+\beta}(\log \log n)^{-1+\beta} \frac{\xi_{n}(1)}{\left[\eta_{n}(1)\right]^{\beta}} \\
& \quad=\frac{\sigma_{f}}{\pi(g)^{\beta}} \sqrt{\frac{2(1-2 \beta)^{(1-2 \beta)(1-p)} \Gamma(p+1)^{1-2 \beta}}{p^{p(1-2 \beta)}(1+(1-2 \beta)(1-p))^{1+(1-2 \beta)(1-p)}}} \text { a.s. }
\end{aligned}
$$

where we adopt the convention that $0^{0}=1$ as $\beta=1 / 2$.

Remark 5.2. The LIL given in (5.2) can be viewed as a connection between the self-normalized LIL $(\beta=1 / 2)$

$$
\limsup _{n \rightarrow \infty} \xi_{n}(1) / \sqrt{2 \eta_{n}(1) \log \log n}=\frac{\sigma_{f}}{\sqrt{\pi(g)}} \text { a.s., }
$$

a form close to those given in Chen [7], and the deterministic normalized LIL $(\beta=0)$

$$
\limsup _{n \rightarrow \infty} \xi_{n}(1) / \sqrt{a\left(\frac{n}{\log \log n}\right)} \log \log n=\sigma_{f} \sqrt{\frac{2 \Gamma(p+1)}{p^{p}(2-p)^{2-p}}} \quad \text { a.s. }
$$

which is obtained in Chen [9].

Proof of Theorem 5.1. First we claim that for any $(x, y) \in \mathcal{X} \equiv(-\infty, \infty) \times[0, \infty)$,

$$
\Lambda_{p}^{*}(x, y)=\inf \left\{I_{p}(\phi, \gamma) ; \phi(1)=x \text { and } \gamma(1)=y\right\} .
$$

Indeed, for any $(\phi, \gamma) \in \Theta_{p}$ with $\phi(1)=x$ and $\gamma(1)=y$, by Jensen's inequality,

$$
\int_{0}^{1} \Lambda_{p}^{*}(\dot{\phi}(t), \dot{\gamma}(t)) d t \geqslant \Lambda_{p}^{*}\left(\int_{0}^{1} \dot{\phi}(t) d t, \int_{0}^{1} \dot{\gamma}(t) d t\right)=\Lambda_{p}^{*}(x, y) .
$$

On the other hand, the equality holds if $\phi(t)=x t$ and $\gamma(t)=y t$.

By (5.3),

$$
\left\{(\phi(1), \gamma(1)) ; I_{p}(\phi, \gamma) \leqslant 1\right\}=\left\{(x, y) ; \Lambda^{*}(x, y) \leqslant 1\right\} .
$$

Hence (5.1) follows from Theorem 4.1.

Let $\rho$ be the constant given on the right hand side of (5.2). By simple calculus,

$$
\sup _{(x, y) \in K\left(\Lambda_{p}^{*}\right)} \frac{x}{y^{\beta}}=\sup \left\{\frac{x}{y^{\beta}} ; \Lambda_{p}^{*}(x, y)=1\right\}=\rho .
$$

Further, one can find $\left(x_{o}, y_{o}\right)$ such that $\Lambda_{p}^{*}\left(x_{o}, y_{o}\right)=1$ and $x_{o} / y_{o}^{\beta}=\rho$. Although the function $\varphi(x, y)=x / y^{\beta}$ is not continuous on $\mathcal{X}$, it is continuous in a small neighborhood of $\left(x_{o}, y_{o}\right)$. By (5.1), therefore,

$$
\limsup _{n \rightarrow \infty} \varphi\left(\left(\Xi_{n}(1)\right) \geqslant \varphi\left(x_{o}, y_{o}\right)=\rho \quad\right. \text { a.s. }
$$

Or,

$$
\limsup _{n \rightarrow \infty} a\left(\frac{n}{\log \log n}\right)^{-\frac{1}{2}+\beta}(\log \log n)^{-1+\beta} \frac{\xi_{n}(1)}{\left[\eta_{n}(1)\right]^{\beta}} \geqslant \rho \text { a.s. }
$$

On the other hand, for given $\varepsilon>0$, define the set

$$
A=\left\{(x, y) \in \mathcal{X} ; x \geqslant(\rho+\varepsilon) y^{\beta}\right\} .
$$


Since $A$ is closed, and by (5.4) $A$ and $K\left(\Lambda_{p}^{*}\right)$ are disjoint, there is a continuous function $\psi: \mathcal{X} \rightarrow[0,1]$ such that $\psi(A)=1$ and $\psi\left(K\left(\Lambda_{p}^{*}\right)\right)=0$. From $(5.1)$,

$$
\limsup _{n \rightarrow \infty} I_{A}\left(\Xi_{n}\right) \leqslant \limsup _{n \rightarrow \infty} \psi\left(\Xi_{n}\right)=\sup _{(x, y) \in K\left(\Lambda_{p}^{*}\right)} \psi(x, y)=0 \quad \text { a.s. }
$$

Hence we have

$$
\limsup _{n \rightarrow \infty} a\left(\frac{n}{\log \log n}\right)^{-\frac{1}{2}+\beta}(\log \log n)^{-1+\beta} \frac{\xi_{n}(1)}{\left[\eta_{n}(1)\right]^{\beta}} \leqslant \rho+\varepsilon \quad \text { a.s. }
$$

Since $\varepsilon>0$ can be arbitrarily small

$$
\limsup _{n \rightarrow \infty} a\left(\frac{n}{\log \log n}\right)^{-\frac{1}{2}+\beta}(\log \log n)^{-1+\beta} \frac{\xi_{n}(1)}{\left[\eta_{n}(1)\right]^{\beta}} \leqslant \rho \quad \text { a.s. }
$$

Let $F$ be an absolutely continuous function $F$ on $[0,1]$ and consider the linear continuous function $\psi$ on $C[0,1]$ :

$$
\psi(\phi)=F(1) \phi(1)-\int_{0}^{1} \phi(t) \cdot F^{\prime}(t) d t, \quad \phi \in C[0,1] .
$$

By integration by parts and Hölder inequality one can see that

$$
\sup _{\phi \in K\left(\kappa_{p}\right)} \psi(\phi)=\sigma_{f} \sqrt{\frac{2 \Gamma(p+1)}{p^{p}(2-p)^{2-p}}}\left(\int_{0}^{1}|F(t)|^{2 / p} d t\right)^{p / 2}
$$

and that if $F$ is non-negative,

$$
\sup _{\phi \in K\left(J_{p}\right)} \psi(\phi)=\frac{\Gamma(p+1) \pi(g)}{p^{p}(1-p)^{1-p}}\left(\int_{0}^{1} F^{1 / p}(t) d t\right)^{p} .
$$

On the other hand, by integration by parts,

$$
\begin{aligned}
& \psi\left(\xi_{n}^{c}\right)=n \sum_{k=1}^{n} f\left(X_{k}\right) \int_{\frac{k-1}{n}}^{\frac{k}{n}} F(t) d t \sim \sum_{k=1}^{n} F\left(\frac{k}{n}\right) f\left(X_{k}\right), \quad n \rightarrow \infty, \\
& \psi\left(\eta_{n}^{c}\right)=n \sum_{k=1}^{n} g\left(X_{k}\right) \int_{\frac{k-1}{n}}^{\frac{k}{n}} F(t) d t \sim \sum_{k=1}^{n} F\left(\frac{k}{n}\right) g\left(X_{k}\right), \quad n \rightarrow \infty,
\end{aligned}
$$

where $\xi_{n}^{c}$ and $\eta_{n}^{c}$ are the continuous versions (Remark 1.5) of $\xi_{n}$ and $\eta_{n}$, respectively.

Therefore, we have the following theorem:

Theorem 5.3. Assume that the Harris recurrent Markov chain $\left\{X_{n}\right\}_{n \geqslant 0}$ is p-regular and that $f$ is a charge, $g$ is special. Let the function $F(t)$ be absolutely continuous on $[0,1]$.

(1) When $0<p<1$ and $F$ is non-negative, 


$$
\begin{gathered}
\limsup _{n \rightarrow \infty} \sum_{k=1}^{n} F\left(\frac{k}{n}\right) g\left(X_{k}\right) / a\left(\frac{n}{\log \log n}\right) \log \log n \\
=\frac{\Gamma(p+1) \pi(g)}{p^{p}(1-p)^{1-p}}\left(\int_{0}^{1} F^{1 / p}(t) d t\right)^{p} \quad \text { a.s. }
\end{gathered}
$$

(2) When $0<p \leqslant 1$,

$$
\begin{gathered}
\limsup _{n \rightarrow \infty} \sum_{k=1}^{n} F\left(\frac{k}{n}\right) f\left(X_{k}\right) / \sqrt{a\left(\frac{n}{\log \log n}\right)} \log \log n \\
\quad=\sigma_{f} \sqrt{\frac{2 \Gamma(p+1)}{p^{p}(2-p)^{2-p}}}\left(\int_{0}^{1}|F(t)|^{2 / p} d t\right)^{p / 2} \text { a.s. }
\end{gathered}
$$

Let $q \geqslant 1$. By considering the continuous functional $\psi$ on $D[0,1]$ :

$$
\psi(\phi)=\int_{0}^{1}|\phi(t)|^{q} d t, \quad \phi \in D[0,1]
$$

we obtain

Theorem 5.4. Assume that the Harris recurrent Markov chain $\left\{X_{n}\right\}_{n \geqslant 0}$ is p-regular and that $f$ is a charge, $g$ is special. Let $q \geqslant 1$.

(1) When $0<p<1$,

$$
\begin{aligned}
& \limsup _{n \rightarrow \infty} \sum_{k=1}^{n} \eta_{k}^{q}(1) / n a^{q}\left(\frac{n}{\log \log n}\right)(\log \log n)^{q} \\
& \quad=(1+p q)^{-1}\left(\frac{1+p q}{1-p}\right)^{q(1-p)}\left(\frac{\pi(g) q^{p} \Gamma\left(p+\frac{1}{q}\right)}{\Gamma\left(\frac{1}{q}\right)}\right)^{q} \text { a.s. }
\end{aligned}
$$

(2) When $0<p \leqslant 1$,

$$
\begin{aligned}
& \limsup _{n \rightarrow \infty} \sum_{k=1}^{n}\left|\xi_{k}(1)\right|^{q} / n a^{q}\left(\frac{n}{\log \log n}\right)(\log \log n)^{q} \\
& \quad=\frac{2}{2+p q}\left(\frac{2+p q}{p(2-p)}\right)^{\frac{q(2-p)}{2}}\left(\frac{\sigma_{f} \Gamma\left(\frac{p}{2}+\frac{1}{q}\right) \sqrt{2 q^{p} \Gamma(p+1)}}{\Gamma\left(\frac{p}{2}\right) \Gamma\left(\frac{1}{q}\right)}\right)^{q} \text { a.s. }
\end{aligned}
$$

Proof. It remains to prove

$$
\begin{aligned}
& \sup _{\gamma \in K\left(J_{p}\right)} \int_{0}^{1} \gamma^{q}(t) d t=(1+p q)^{-1}\left(\frac{1+p q}{1-p}\right)^{q(1-p)}\left(\frac{\pi(g) q^{p} \Gamma\left(p+\frac{1}{q}\right)}{\Gamma\left(\frac{1}{q}\right)}\right)^{q}, \\
& \sup _{\gamma \in K\left(\kappa_{p}\right)} \int_{0}^{1}|\gamma(t)|^{q} d t=\frac{2}{2+p q}\left(\frac{2+p q}{p(2-p)}\right)^{\frac{q(2-p)}{2}}\left(\frac{\sigma_{f} \Gamma\left(\frac{p}{2}+\frac{1}{q}\right) \sqrt{2 q p \Gamma(p+1)}}{\Gamma\left(\frac{p}{2}\right) \Gamma\left(\frac{1}{q}\right)}\right)^{q} .
\end{aligned}
$$


We now evaluate the variation in (5.9). The idea is the Lagrange multiplier, which has been used in Strassen [32] for the application of his well known invariance principle. Since $K\left(J_{p}\right)$ is compact in $C[0,1]$, the supremum can be achieved at some $\gamma \in K\left(J_{p}\right)$. Fix a such function $\gamma$. One can easily see that $\gamma$ satisfies the equation

$$
(1-p) \int_{0}^{1}\left(\frac{p^{p} \dot{\beta}(t)}{\pi(g) \Gamma(p+1)}\right)^{(1-p)^{-1}} d t=1 .
$$

Notice that the supremum given in (5.9) is also the supremum of the same functional over the set of functions $\beta \in C[0,1]$ satisfying (5.11) with $\beta(0)=0(\beta(t)$ does not have to be non-decreasing or non-negative).

Applying the Lagrange multiplier one can see that there is a real number $\lambda$ such that for all such $\beta$,

$$
q \int_{0}^{1} \gamma^{q-1}(t) \beta(t) d t=\lambda \frac{1}{1-p} \int_{0}^{1} \dot{\gamma}^{p(1-p)^{-1}}(t) \dot{\beta}(t) d t .
$$

Performing integration by parts on the left hand side gives

$$
q \int_{0}^{1}\left[\int_{t}^{1} \gamma^{q-1}(s) d s\right] \dot{\beta}(t) d t=\lambda \frac{1}{1-p} \int_{0}^{1} \dot{\gamma}^{p(1-p)^{-1}}(t) \dot{\beta}(t) d t .
$$

Hence, we must have

$$
q \int_{t}^{1} \gamma^{q-1}(s) d s=\lambda \frac{1}{1-p} \dot{\gamma}^{p(1-p)^{-1}}(t) .
$$

In particular, $\gamma$ has continuous second derivative, $\dot{\gamma}(t)>0$ as $0 \leqslant t<1$ and $\dot{\gamma}(1)=0$. Taking derivative on the both sides of the above equation gives

$$
-q \gamma^{q-1}(t)=\lambda \frac{p}{(1-p)^{2}}(\dot{\gamma}(t))^{\frac{2 p-1}{1-p}} \ddot{\gamma}(t) .
$$

Multiplying both sides by $\dot{\gamma}$ and integrating we obtain

$$
\gamma^{q}(t)=-\lambda \frac{p}{1-p}\left[\dot{\gamma}^{(1-p)^{-1}}(t)-\dot{\gamma}^{(1-p)^{-1}}(0)\right] .
$$

Recall that $\dot{\gamma}(1)=0$. As $t=1$,

$$
\gamma^{q}(1)=\lambda \frac{p}{1-p} \dot{\gamma}^{(1-p)^{-1}}(0) .
$$

Therefore,

$$
\lambda \frac{p}{1-p} \dot{\gamma}^{(1-p)^{-1}}(t)=\gamma^{q}(1)-\gamma^{q}(t) .
$$

Integrating both side of (5.12) and taking (5.11) into account we have

$$
\lambda \frac{p}{(1-p)^{2}}\left(\frac{\pi(g) \Gamma(p+1)}{p^{p}}\right)^{(1-p)^{-1}}=\gamma^{q}(1)-\int_{0}^{1} \gamma^{q}(t) d t .
$$

From (5.12),

$$
d t=\left(\frac{\lambda p}{1-p}\right)^{1-p}\left(\gamma^{q}(1)-\gamma^{q}(t)\right)^{p-1} d \gamma(t) .
$$


Hence,

$$
\begin{aligned}
\int_{0}^{1} \gamma^{q}(t) d t & =\left(\frac{\lambda p}{1-p}\right)^{1-p} \int_{0}^{\gamma(1)} \gamma^{q}\left(\gamma^{q}(1)-\gamma^{q}\right)^{p-1} d \gamma \\
& =\left(\frac{\lambda p}{1-p}\right)^{1-p} \gamma(1)^{1+p q} \int_{0}^{1} u^{q}\left(1-u^{q}\right)^{p-1} d u \\
& =\left(\frac{\lambda p}{1-p}\right)^{1-p} \gamma(1)^{1+p q} \frac{1}{1+p q} \int_{0}^{1}\left(1-u^{q}\right)^{p-1} d u
\end{aligned}
$$

where the last step follows from integration by parts. Similarly,

$$
\begin{aligned}
1 & =\left(\frac{\lambda p}{1-p}\right)^{1-p} \int_{0}^{\gamma(1)}\left(\gamma^{q}(1)-\gamma^{q}\right)^{p-1} d \gamma \\
& =\left(\frac{\lambda p}{1-p}\right)^{1-p} \gamma(1)^{1+q(p-1)} \int_{0}^{1}\left(1-u^{q}\right)^{p-1} d u .
\end{aligned}
$$

Combining (5.13), (5.14) and (5.15) gives

$$
\begin{aligned}
\int_{0}^{1} \gamma^{q}(t) d t & =(1+p q)^{-1}\left(\frac{1+p q}{q(1-p)}\right)^{q(1-p)}\left(\frac{\pi(g) \Gamma(p+1)}{p}\right)^{q}\left(\int_{0}^{1}\left(1-u^{q}\right)^{p-1} d u\right)^{-q} \\
& =(1+p q)^{-1}\left(\frac{1+p q}{1-p}\right)^{q(1-p)}\left(\frac{\pi(g) q^{p} \Gamma\left(p+\frac{1}{q}\right)}{\Gamma\left(\frac{1}{q}\right)}\right)^{q} .
\end{aligned}
$$

The proof of (5.10) is similar to that of (5.19), for the maximizer of the variation in (5.10) is a non-negative function.

\section{Acknowledgements}

The first author is grateful to Professor Alejandro de Acosta for some stimulating discussions which motivated this work. The second author wants to thank Professor Liming Wu for his well pointed remarks.

\section{References}

[1] A. de Acosta, Large deviations for vector-valued functional of Markov chain: lower bounds, Ann. Probab. 16 (1988) 925-960.

[2] A. de Acosta, Moderate deviations for empirical measures of Markov chains: lower bounds, Ann. Probab. 25 (1997) 259-284.

[3] A. de Acosta, X. Chen, Moderate deviations for empirical measures of Markov chains: upper bounds, J. Theoret. Probab. 11 (1998) 1075-1110.

[4] K.B. Athreya, P. Ney, A new approach to the limit theory of recurrent Markov chains, Trans. Amer. Math. Soc. 245 (1978) $493-501$.

[5] J. Azema, M. Duflo, D. Revuz, Propriétés relatives des processus de Markov récurrents, Z. Wahr. Geb. 13 (1969) $286-314$.

[6] X. Chen, Limit theorems for functionals of ergodic Markov chains with general state space, Mem. Amer. Math. Soc. 139 (664) (1999). 
[7] X. Chen, The law of the iterated logarithm for functionals of Harris recurrent Markov chains: self normalization, J. Theoret. Probab. 12 (1999) 421-445.

[8] X. Chen, How often does a Harris recurrent Markov chain recur?, Ann. Probab. 27 (1999) 1324-1346.

[9] X. Chen, On the limit laws of the second order for additive functionals of Harris recurrent Markov chains, Probab. Theory Related Fields 116 (2000) 89-123.

[10] X. Chen, Moderate deviations for Markovian occupation times, Stochastic Process. Appl. 94 (2001) 51-70.

[11] K.L. Chung, G.A. Hunt, On the zeros of $\sum_{1}^{n} \pm 1$, Ann. of Math. 50 (1949) 385-400.

[12] E. Csáki, M. Csörgö, On additive functionals of Markov chains, J. Theoret. Probab. 8 (1995) $905-919$.

[13] E. Csáki, P. Salminen, On the additive functionals of diffussion processes, Studia Sci. Math. Hungar. 31 (1996) $47-62$.

[14] A. Dembo, Q. Shao, Self-normalized moderate deviations and LILs, Stochastic Process. Appl. 75 (1998) 51-65.

[15] A. Dembo, O. Zeitouni, Large Deviations Techniques and Applications, Jones and Bartlett, Boston, 1993.

[16] H. Djellout, A. Guillin, L. Wu, Large and moderate deviations for quadratic empirical processes, Stat. Inf. Stoch. Proc. 2 (1999) $195-225$.

[17] H. Djellout, A. Guillin, Moderate deviations of Markov Chains with atom, Stochastic Process. Appl. 95 (2001) $203-217$.

[18] M. Duflo, Random Iterative Models, Springer, New York, 1997.

[19] P. Erdös, S.J. Taylor, Some problems concerning the structure of random walks, Acta Math. 11 (1960) $137-162$.

[20] N. Gantert, O. Zeitouni, Large and moderate deviations for the local time of a recurrent Markov chain on $Z^{2}$, Ann. Inst. H. Poinc. (Probab. Statist.) 34 (1998) 687-704.

[21] A. Guillin, Uniform moderate deviations of functional empirical processes of Markov chains, Probab. Math. Stat. 20 (2000) 237-260.

[22] N.C. Jain, W.E. Pruitt, Asymptotic behavior for the local time of a recurrent random walk, Ann. Probab. 11 (1983) 64-85.

[23] T. Jiang, M.B. Rao, X. Wang, D. Li, Laws of large numbers and moderate deviations for stochastic processes with stationary and independent increments, Stochastic Process. Appl. 44 (1993) 205-219.

[24] H. Kesten, An iterated logarithm law for local times, Duke Math. J. 32 (1965) 447-456.

[25] J.-F Le Gall, J. Rosen, The range of stable random walks, Ann. Probab. 19 (1991) 650-705.

[26] S.P. Meyn, R.L. Tweedie, Markov Chains and Stochastic Stability, Springer-Verlag, London, 1993.

[27] M.B. Marcus, J. Rosen, Law of the iterated logarithm for the local times of symmetric Lévy processes and recurrent random walks, Ann. Probab. 22 (1994) 626-658.

[28] E. Nummelin, A splitting technique for Harris recurrent chains, Z. Wahr. Geb. 43 (1978) 309-318.

[29] E. Nummelin, General Irreducible Markov Chains and Non-negative Operators, Cambridge University Press, Cambridge, 1984.

[30] S. Orey, Limit Theorems for Markov Chain Transition Probabilities, Van Nostrand Reinhold, London, 1971.

[31] P. Révész, Random Walk in Random and Non-random Environments, World Scientific, London, 1990.

[32] V. Strassen, An invariance principle for the law of the iterated logarithm, Z. Wahr. Geb. 3 (1964) 211-226.

[33] D. Revuz, Markov Chains, North-Holland, New York, 1975.

[34] A. Touati, Loi fonctionnelle du logarithme itéré pour les processus de Markov récurrents, Ann. Probab. 18 (1990) $140-159$.

[35] L. Wu, Moderate deviations for random variables related to CLT, Ann. Probab. 23 (1995) 420-445. 